\title{
RESEARCH
}

Open Access

\section{Expression profiling of Trypanosoma congolense genes during development in the tsetse fly vector Glossina morsitans morsitans}

Erick O. Awuoche ${ }^{1,2,3,4^{*}}$, Brian L. Weiss ${ }^{3}$, Paul O. Mireji ${ }^{1,3,5}$, Aurélien Vigneron ${ }^{3}$, Benson Nyambega ${ }^{6}$, Grace Murilla' and Serap Aksoy ${ }^{3}$

\begin{abstract}
Background: The tsetse transmitted parasitic flagellate Trypanosoma congolense causes animal African trypanosomosis (AAT) across sub-Saharan Africa. AAT negatively impacts agricultural, economic, nutritional and subsequently, health status of the affected populace. The molecular mechanisms that underlie T. congolense's developmental program within tsetse are largely unknown due to considerable challenges with obtaining sufficient parasite cells to perform molecular studies.

Methods: In this study, we used RNA-seq to profile T. congolense gene expression during development in two distinct tsetse tissues, the cardia and proboscis. Indirect immunofluorescent antibody test (IFA) and confocal laser scanning microscope was used to localize the expression of a putative protein encoded by the hypothetical protein (TClL3000_0_02370).

\footnotetext{
*Correspondence: otieno43@gmail.com

'Department of Biochemistry, Biotechnology Research Institute, Kenya

Agricultural and Livestock Research Organization, Kikuyu, Kenya

${ }^{2}$ Department of Biomedical Science and Technology, School of Public Health

and Community Development, Maseno University, Private Bag, Maseno, Kenya

Full list of author information is available at the end of the article
}

(c) The Author(s). 2018 Open Access This article is distributed under the terms of the Creative Commons Attribution 4.0 International License (http://creativecommons.org/licenses/by/4.0/), which permits unrestricted use, distribution, and reproduction in any medium, provided you give appropriate credit to the original author(s) and the source, provide a link to the Creative Commons license, and indicate if changes were made. The Creative Commons Public Domain Dedication waiver (http://creativecommons.org/publicdomain/zero/1.0/) applies to the data made available in this article, unless otherwise stated. 
(Continued from previous page)

Results: Consistent with current knowledge, genes coding several variant surface glycoproteins (including metacyclic specific VSGs), and the surface coat protein, congolense epimastigote specific protein, were upregulated in parasites in the proboscis (PB-parasites). Additionally, our results indicate that parasites in tsetse's cardia (C-parasites) and PB employ oxidative phosphorylation and amino acid metabolism for energy. Several genes upregulated in C-parasites encoded receptor-type adenylate cyclases, surface carboxylate transporter family proteins (or PADs), transport proteins, RNA-binding proteins and procyclin isoforms. Gene ontology analysis of products of genes upregulated in C-parasites showed enrichment of terms broadly associated with nucleotides, microtubules, cell membrane and its components, cell signaling, quorum sensing and several transport activities, suggesting that the parasites colonizing the cardia may monitor their environment and regulate their density and movement in this tissue. Additionally, cell surface protein (CSP) encoding genes associated with the Fam50 'GARP', 'iii' and 'i' subfamilies were also significantly upregulated in C-parasites, suggesting that they are important for the long non-dividing trypomastigotes to colonize tsetse's cardia. The putative products of genes that were upregulated in PB-parasites were linked to nucleosomes, cytoplasm and membrane-bound organelles, which suggest that parasites in this niche undergo cell division in line with prior findings. Most of the CSPs upregulated in PB-parasites were hypothetical, thus requiring further functional characterization. Expression of one such hypothetical protein (TcIL3000_0_02370) was analyzed using immunofluorescence and confocal laser scanning microscopy, which together revealed preferential expression of this protein on the entire surface coat of T. congolense parasite stages that colonize G. m. morsitans' proboscis.

Conclusion: Collectively, our results provide insight into T. congolense gene expression profiles in distinct niches within the tsetse vector. Our results show that the hypothetical protein TCIL3000_0_02370, is expressed on the entire surface of the trypanosomes inhabiting tsetse's proboscis. We discuss our results in terms of their relevance to disease transmission processes.

Keywords: Trypanosoma congolense, Glossina morsitans morsitans, Gene expression analysis, Tsetse cardia, Tsetse proboscis and confocal microscopy

\section{Background}

Tsetse (Glossina spp.)-transmitted Trypanosoma congolense is a major cause of animal African trypanosomosis (AAT) in livestock in most of sub-Saharan Africa. The disease has a significant economic and public health impact due to its wide geographical distribution and broad vertebrate animal host range $[1,2]$. To date no vaccines against $T$. congolense exist. Thus, AAT management relies on controlling the tsetse vector via use of baited traps and targets, spraying with insecticides and/or treatment of infected animals using trypanocidal chemotherapy. Inherent limitations underlying successful application of these strategies include drug resistance in the parasite [3-7] and re-infestation by residual tsetse populations where and when control programs are abandoned [8]. These limitations necessitate development of novel intervention strategies that may interfere with parasite transmission through the tsetse vector. Accordingly, additional information on genetic factors that regulate $T$. congolense development in the tsetse fly is required.

African trypanosomes ( $T$. congolense, $T$. vivax and $T$. brucei) must infect vertebrate hosts and tsetse vectors in order to complete their life-cycle. To do so the parasites must progress through a series of developmental forms adapted to each life-cycle stage. Vertebrate infectious metacyclic T. congolense and T. vivax develop in tsetse's proboscis $(\mathrm{PB})$ while metacyclic $T$. brucei form in the fly's salivary glands (SG). In the mammalian host, $T$. congolense proliferates as single type of bloodstream form (BSF) parasite covered with carbohydrate-rich variant surface glycoproteins (VSGs) [9]. Upon entry into the lumen of tsetse's gut, BSF $T$. congolense differentiate to procyclic forms (PCF), and this transformation is accompanied by changes in parasite morphology, a switch from glycolysis to oxidative phosphorylation for energy metabolism and replacement of the VSG surface coat with one composed of procyclins [10]. In successful infections, the parasites migrate to tsetse's cardia, transform into long non-dividing trypomastigote parasites and subsequently migrate to and colonize the fly's PB. Finally, these cells differentiate into epimastigote forms (EMF), which attach to the PB wall and give rise to mammalian infective metacyclic forms (MCFs) $[11,12]$. Molecular factors that regulate differentiation of $T$. congolense, and major barriers to T. congolense transmission through the tsetse vector, are poorly understood. Sequencing and annotation of the genomes of the three African trypanosomes and six species of tsetse fly, along with advances in RNA sequencing (RNA-seq) methodologies, have significantly expanded our knowledge of the molecular biology that underlies this vector-pathogen system. However, most research to date has been performed using $T$. brucei [13-22], with two RNA-seq and one proteomic study reported in $T$. vivax $[23,24]$ and a single transcriptomic and proteomic analysis performed with in vitro cultured $T$. congolense cells $[25,26]$. 
In this study, we utilized high throughput RNA-seq to profile expression of $T$. congolense genes in infected cardia and PB organs of G. morsitans morsitans. We mined the data to identify molecular factors that may be functionally involved in vector-parasite interactions, parasite differentiation and maintenance of $T$. congolense in tsetse's cardia and PB. We established that $15.95 \%$ of all genes were expressed at significantly different levels in C-parasites compared to PB-parasites. More specifically, genes upregulated in $\mathrm{C}$-parasites encoded transporter and Fam50 ('GARP, 'iii' and 'i' subfamily) proteins, while those upregulated in PB-parasites encoded VSGs among other proteins associated with parasite multiplication. We discuss our findings in the context of furthering new initiatives for development of novel disease control applications.

\section{Methods}

\section{Trypanosome strain, tsetse flies and tsetse infection}

Trypanosoma congolense [Trans Mara strain, variant antigenic type (VAT) TC13] used in this study were kindly provided by Professor Utpal Pal, at Department of Veterinary Medicine, University of Maryland. The parasite was originally isolated from an infected bovine in Trans Mara, Kenya [27]. The VAT TC13 was obtained by serial cloning of the parasite in immunosuppressed CD1 mice [28]. The BSF TC13 was amplified in rats and harvested from blood at peak parasitemia by cardiac puncture.

Tsetse flies (Glossina morsitans morsitans) used in this study were reared in Yale University (New Haven, CT, USA) insectary at $24{ }^{\circ} \mathrm{C}$ and $50 \%$ relative humidity. In this colony, tsetse flies are fed at 48 hour intervals using an artificial membrane-based feeding system [29] with defibrinated bovine blood commercially supplied by Hemostat Laboratories (Dixon, CA, USA). Teneral (newly eclosed and unfed) G. $m$. morsitans males were infected by feeding them a blood meal supplemented with BSF $T$. congolense (VAT TC13) $\left(8 \times 10^{6}\right.$ parasites per $\mathrm{ml}$ of blood via artificial membrane feeding method [29]). Subsequently, the flies were maintained on normal blood meal for 28 days until tissue dissection was performed.

\section{Dissection and collection of tsetse tissues}

The cardia and probosces of $T$. congolense infected flies were dissected 28 days post-challenge and $72 \mathrm{~h}$ after the last blood meal. Dissections of cardia for T. congolense infection analyses were microscopically conducted in PSG buffer ( $\mathrm{pH}$ 8.0) using Zeiss Axiostar Plus light microscope (Carl Zeiss Light Microscopy, Gottingen) at $\times 400$ magnification. Two biological replicates of infected probosces (consisting of 130 probosces each) from a recent study were used [30]. Infected cardia were immediately placed in TRIzol (Thermo Fisher Scientific Inc., CA, USA). Three biological replicates consisting of 15 infected cardia each were used. Only flies which had both cardia and proboscis infections were used for all downstream analyses.

RNA extraction, cDNA library preparation and sequencing Total RNA extraction and subsequent elimination of contaminating DNA was done using TRIzol and Turbo-DNase (Thermo Fisher Scientific Inc., CA, USA), respectively, following the manufacturer's protocol. Elimination of DNA from the total RNA was confirmed by PCR using both T. congolense- and G. m. morsitansspecific beta-tubulin and glyceraldehyde-3-phosphate dehydrogenase (gapdh) primers for trypanosome and tsetse fly, respectively. RNA quantity and quality were determined using a Bioanalyzer 2100 (Agilent, Palo Alto, CA, USA) and subsequent cDNA libraries prepared using a NEBNext Ultra Directional RNA Library Prep Kit (New England Biolabs, Inc., MA, USA) according to the manufacturer's protocol. The cardia and probosces libraries were barcoded for Illumina HiSeq 2000 sequencing (Illumina, Inc., CA, USA) (unpaired 75 bases) at Yale Center for Genome Analysis (YCGA, New Haven, $\mathrm{CT})$. For the generation of these cDNA libraries, $900 \mathrm{ng}$ of high quality (RNA integrity number > 7.0) total RNA samples was used. The Sequence Read Archive number at NCBI of infected cardia is SRP093558 and that of infected probosces is PRJNA354110 [30].

\section{Processing and differential analyses of $T$. congolense transcriptome}

All bioinformatics analyses were conducted using CLC Genomics Workbench version 8.5 (CLC bio, Cambridge, MA). The fasta RNA-seq data were first processed for quality conformity and then mapped to $T$. congolense IL 3000 reference strain transcripts version 9 [31] obtained from TritrypDB [32]. The TC13 strain used in this study was different from the strain from which the whole genome data were generated, but both parasite strains had originated from Trans Mara in Kenya [33]. The mapping employed algorithms that allowed for only two mismatches and a maximum of 10 hits per read with at least $80 \%$ of the reads matching the gene at $95 \%$. Reads per kilobase per million mapped (RPKM) [34] was used as a proxy of gene expression. Differentially expressed (DE) genes were determined using RNA-Seq module employing Baggeley's test and Bonferroni analysis [35]. Relative fold change (FC) between parasite genes from the cardia and proboscis was calculated as a ratio of their respective RPKM values. Differential expression analyses were also conducted using EdgeR software [36] to corroborate the results obtained by CLC Genomics Workbench. A conservative selection regime was adopted to minimize false detections of differential expression. Within this 
regime, transcripts were considered $\mathrm{DE}$ between parasites in cardia and probosces if they had (i) at least two-FC in either of the tools used (CLC-genomics or EdgeR), (ii) normalized false detection rate (FDR) corrected $P$-value of $\leq 0.05,3$ ) supported by at least 30 reads mapping in either library (C-parasite or PB-parasite), (iv) at least five RPKM, and (v) considered as DE with both tools (CLC-genomics and EdgeR).

\section{Functional annotation of DE genes}

Functional annotations of DE genes were conducted using Blast2GO version 3.4 program [37-39]. Briefly, homology searches for all $T$. congolense genes were conducted against NCBI non-redundant (nr) protein database using BLASTx [40] with an E-value BLAST cut-off of 1.0E-03. The blast results were then mapped and annotated for gene ontology $(\mathrm{GO})$ categories related to biological processes, molecular function and cell component using an algorithm in Blast2GO software. Enriched GO terms among DE gene products were established by Fisher's exact test at a false discovery rate (FDR) $P$-value $\leq 0.05$ [38]. Protein domains/signatures associated with putative products of the DE genes were determined through InterProScan analysis [41]. The DE genes encoding proteins with predicted cell-surface functions were retrieved from previously published data [42] and validated using PredGIP [43], FragAnchor [44] and BigPI [45] softwares for GPI-anchor motifs prediction, and TMHMM [46] software for trans-membrane helices prediction. Secreted proteins were predicted using SignalP version 1.4 software [47]. Enriched metabolic pathways for gene products were identified using KEGG [48, 49] and TrypanoCyc [50] tools implemented in TritrypDB at $\mathrm{FDR} \leq 0.05$.

\section{Real time quantitative-PCR (RT-qPCR) analysis for transcriptome validation}

RT-qPCR analysis was conducted on independent samples to corroborate RNA-seq data. Samples for transcriptome validation were obtained from an independent set of $T$. congolense infected tsetse cardia or probosces. Total RNA and DNase treatment of samples of infected cardia or probosces were prepared as described above. Eight biological replicates of infected cardia or probosces were used, each containing a pool of 5 and 25 tissues, respectively. cDNA was synthesized with oligo-dT primers and random hexamers using iScript cDNA synthesis reaction kit (Bio-Rad, $\mathrm{CA}$ ) according to the manufacturer's protocol. RT-qPCR was performed in technical duplicate (for each biological replicate) on eight selected DE genes (Additional file 1: Text S1). All RT-qPCR results were normalized using $T$. congolense gapdh (TcIL3000_10_5910) and 60S ribosomal (TcIL3000_0_32580) genes determined from each biological replicate. The two genes were more stable in these two tissues with standard deviation of the crossing point being less than 1 according to BestKeeper analysis [51]. Correlation between RT-qPCR and RNA-seq fold change results were evaluated using Pearson correlation analysis (Additional file 2: Text S2).

\section{Semi-quantitative RT-PCR analysis of gene expression}

Semi-quantitative RT-PCR was performed for selected cell surface protein (CSP) encoding genes using cDNA samples synthesized from RNA isolated from parasites obtained from infected rats, tsetse midgut, cardia or probosces, following a previously published method [52]. To normalize cDNA samples from various tsetse tissues and BSF for PCR analysis, equal amounts of samples were diluted 10-fold and run for 28 PCR cycles using $T$. congolense specific gapdh primers. The PCR normalization of samples using gapdh was performed at the following cycling conditions: $2 \mathrm{~min}$ at $95{ }^{\circ} \mathrm{C}$ followed by 28 cycles at $95^{\circ} \mathrm{C}$ for $45 \mathrm{~s}, 55^{\circ} \mathrm{C}$ for $1 \mathrm{~min}$ and $72{ }^{\circ} \mathrm{C}$ for $1 \mathrm{~min}$. A final $75{ }^{\circ} \mathrm{C}$ extension was performed for $10 \mathrm{~min}$. For experimental analysis, all PCR reactions were performed as technical replicates as described at 34 cycles for each sample (from infected rats, tsetse midgut, cardia or probosces cDNAs) and the PCR products individually resolved on $1 \%$ agarose gel. As control for experimental analysis, gapdh gene was also amplified at 34 PCR cycles. Gel visualization, image capturing and semi-quantitative measurement of expression variation between different tissue samples was done using Gel Doc ${ }^{\text {tw }} \mathrm{XR}+$ Gel Documentation System (Bio-Rad, CA, USA). The expression values of each gene for each parasite stage were normalized to those of similarly treated gapdh controls. Fold change for each of the three-developmental tsetse tissue-specific and BSF samples was calculated as described by Savage et al. [52]. Primers for each gene used in this analysis can be found in Additional file 1: Text S1.

\section{Recombinant protein expression, purification and antibody production}

The gene encoding a hypothetical Fam50 protein, (TcIL3000_0_02370), was cloned into pET28a expression vector without signal peptide and GPI-anchor domains, sequenced, and expressed in BL21 competent bacterial cells (Promega, Madison, WI). For cloning, BamHI and XhoI enzyme restriction site sequences were added to the forward and reverse primers respectively (Additional file 1: Text S1). For recombinant expression, the putative 224 amino acids (26 to 249 from the N-terminal) was cloned. Recombinant protein expression was induced with $1 \mathrm{mM}$ isopropyl- $\beta$-thiogalactoside at $28{ }^{\circ} \mathrm{C}$, purified using $6 \times$ His-tag pull down and analyzed by polyacrylamide gel electrophoresis for purity. The rec-protein was then concentrated by centrifugation in a PM-membrane of $10 \mathrm{MW}$ cut-off (Thermo Fisher Scientific Inc., CA, 
USA). Recombinant protein yield was determined by BCA kit (PIERCE Chemical Company, Rockford, IL, USA). Polyclonal rabbit antibodies against recombinant TcIL3000_0_02370 protein was commercially generated by Cocalico Biologicals, Inc., PA, USA.

\section{Immunofluorescence assay (IFA) and microscopic observation}

IFA was performed to localize expression of TcIL3000_ 0_02370 CSP. Tsetse-derived T. congolense parasites harvested from infected tsetse midgut, cardia or proboscis organs 28-days post-infections were individually placed in PSG buffer for 5-10 min to enable parasites to diffuse out of the tissues and into the buffer. Parasites in the buffer were then spotted onto a glass slide and cells fixed with $4 \%$ paraformaldehyde at room temperature for $30 \mathrm{~min}$. To permeabilize the samples, slides were placed $10 \mathrm{~min}$ in $0.1 \%$ Triton X-100 PBS and then washed twice in PBS prior to the blocking step. Both permeabilized and non-permeabilized samples were blocked with $5 \%$ bovine serum albumin (BAS) in PBS for $30 \mathrm{~min}$ and then incubated overnight at $4{ }^{\circ} \mathrm{C}$ with rabbit anti-TcIL3000_0_02370 polyclonal antibody diluted 1:100 in 5\% BSA in PBS. Slides were then washed twice for $5 \mathrm{~min}$ in 5\% BSA in PBS at RT prior to being incubated for $1 \mathrm{~h}$ at RT with goat anti-rabbit Alexa596-conjugated secondary antibodies diluted 1:500 in 5\% BSA in PBS. Slides were then washed twice for 5 min in PBS at RT before being quickly washed in ultrapure water to remove excess salts. Slides were air-dried, mounted with VectaShield H-1500, stained with $1 \mu \mathrm{g} / \mathrm{ml}$ DAPI (to visualize the cells' nucleus and kinetoplast) and washed twice with PBS ( $\mathrm{pH}$ 8.0) as described above. Slides were observed with a Zeiss LSM 710 confocal microscope (Zeiss, Germany). Images were merged and contrasts-treated using the module Fiji for the ImageJ software [53].

\section{Results}

\section{Abundance of $T$. congolense transcripts in infected cardia} and proboscis

We performed RNA-seq analyses of $T$. congolense in infected tsetse cardia (C-parasites) and proboscis (PB-parasites) organs and characterized the expression of parasite specific transcripts. Results from the infected tissues revealed that $7.34 \%$ and $4.04 \%$ of the total reads obtained from the infected cardia and proboscis transcriptomes, respectively, mapped to the putative T. congolense IL3000 gene sets. These included genes encoding putative T. congolense VSGs (Additional file 3: Figure S1a). Upon mapping, transcripts were detected for $91.8 \%$ of the 13,549 T. congolense IL3000 genes. Most of these genes (74\% and $66 \%$ of the C- and PB-parasites, respectively) exhibited low transcript abundance ( $\leq 100$ total reads).
An average of $1.12 \%$ of the genes were categorized as having high transcript abundance ( $>10,000$ total reads) in both libraries (Additional file 3: Figure S1b).

We next examined the genes comprising the top 99 percentile (RPKM) in each tissue (C-parasite or PB-parasite) stage to identify the most abundantly expressed genes in each tissue, and assess the extent of overlap. This analysis identified 131 genes, of which 56 were common between $\mathrm{C}$ - and $\mathrm{PB}$-parasites. The remaining 75 genes were in the top RPKM percentile in one of the tissue-specific parasites (Additional file 3: Figure S1c, Additional file 4: Table S1). Gene ontology enrichment analysis of the putative shared gene products showed enriched terms broadly related to ribosome, translation, transport and GTPase activity (Table 1), indicating abundant protein synthesis and transport activity. Metabolic pathway analysis of the shared abundant products identified cysteine and methionine, aspartate superpathway, methionine salvage cycle, glycerol degradation, trypanothine and S-adenosylmethionine biosynthesis, as well as peroxide metabolic pathways, as enriched (Table 1). The methionine cycle recycles methionine from methylthioadenosine, which is a by-product of polyamine synthesis. The activity of this cycle in recycling methionine was previously proposed in T. brucei [54]. However, the methionine cycle is now thought to be inactive in trypanosomes, at least in PCF and BSF T. brucei parasite stages $[55,56]$. The functionality of this pathway remains to be elucidated in other trypanosome stages and species, including $T$. congolense. Degradation of glycerol, a carbon source likely present in insect vectors, and enrichment of the aspartate superhighway, could mean that these two pathways may serve as the source of acetate in lipid biosynthesis. High expression of putative proteins functionally linked with trypanothione and peroxide metabolic pathways may be important for the parasites to survive in conditions under high oxidative stress [57-59] that may exist in infected cardia and probosces.

Of the remaining 75 most abundant transcripts, 33 were present only in the top 1\% RPKM of the C-parasite library. These putatively encoded Glycosomal phosphoenolpyruvate carboxykinase, Zinc finger protein (ZC3H36), Cytochrome oxidase IV, Cysteine peptidase, and several transporter and receptor-type adenylate cyclase proteins. In addition, putative cell surface proteins, such as Procyclin-like and GARP proteins, were most abundant in C-parasites (Additional file 4: Table S1). The remaining genes (42 out of 75 ) were most abundantly expressed in PB-parasites and they included transcripts encoding 23 ribosomal proteins, three histones (H2A, two-H2B), two actins, and several cell surface proteins (Additional file 4: Table S1). Thus, the PB-parasite data imply an increased rate of protein synthesis at this stage, which is an important phenomenon during active cell division. 
Table 1 Gene Ontology and metabolic enriched pathways of the 99-percentile common most highly expressed gene products

\begin{tabular}{|c|c|c|c|c|c|c|}
\hline \multicolumn{7}{|c|}{ Gene ontology enriched pathway } \\
\hline Category & GO ID & Term & FDR & $P$-value & Test $^{\mathrm{a}}$ & $\operatorname{Ref}^{b}$ \\
\hline \multirow[t]{3}{*}{ Cell component } & GO:0005840 & Ribosome & 7.98E-09 & 3.56E-12 & 15 & 172 \\
\hline & GO:0022627 & Cytosolic small ribosomal subunit & 1.77E-04 & 5.93E-07 & 3 & 3 \\
\hline & GO:0000314 & Organellar small ribosomal subunit & 4.84E-02 & 7.12E-04 & 2 & 5 \\
\hline \multirow[t]{9}{*}{ Molecular function } & GO:0003735 & Structural constituent of ribosome & 1.89E-08 & $1.26 \mathrm{E}-11$ & 14 & 154 \\
\hline & GO:0015114 & Phosphate ion transmembrane transporter activity & 9.55E-03 & 7.24E-05 & 2 & 2 \\
\hline & GO:0005315 & Inorganic phosphate transmembrane transporter activity & 9.55E-03 & 7.24E-05 & 2 & 2 \\
\hline & GO:0003924 & GTPase activity & 1.35E-02 & $1.11 \mathrm{E}-04$ & 5 & 57 \\
\hline & GO:0004478 & Methionine adenosyltransferase activity & 2.11E-02 & $2.16 \mathrm{E}-04$ & 2 & 3 \\
\hline & GO:0019843 & rRNA binding & 2.87E-02 & 3.07E-04 & 3 & 16 \\
\hline & GO:0005515 & Protein binding & 2.92E-02 & 3.19E-04 & 10 & 325 \\
\hline & GO:0005200 & Structural constituent of cytoskeleton & $3.33 \mathrm{E}-02$ & $3.71 \mathrm{E}-04$ & 3 & 17 \\
\hline & GO:0015224 & Biopterin transporter activity & 3.33E-02 & 4.30E-04 & 2 & 4 \\
\hline \multirow[t]{8}{*}{ Biological processes } & GO:0006412 & Translation & 9.19E-07 & 1.43E-09 & 15 & 261 \\
\hline & GO:0006817 & Phosphate ion transport & 9.55E-03 & $7.24 \mathrm{E}-05$ & 2 & 2 \\
\hline & GO:0000028 & Ribosomal small subunit assembly & 9.55E-03 & 7.24E-05 & 2 & 2 \\
\hline & GO:0006915 & Apoptotic process & 1.67E-02 & 1.60E-04 & 3 & 13 \\
\hline & GO:0006556 & S-adenosylmethionine biosynthetic process & 2.11E-02 & $2.16 \mathrm{E}-04$ & 2 & 3 \\
\hline & GO:0015877 & Biopterin transport & 3.33E-02 & 4.30E-04 & 2 & 4 \\
\hline & GO:0006986 & Response to unfolded protein & 3.33E-02 & 4.30E-04 & 2 & 4 \\
\hline & GO:0051258 & Protein polymerization & 4.35E-02 & $6.11 \mathrm{E}-04$ & 3 & 20 \\
\hline \multicolumn{7}{|l|}{ Metabolic pathways } \\
\hline Database & Pathway ID & Pathway description & FDR & P-value & Test $^{a}$ & $\operatorname{Ref}^{b}$ \\
\hline KEGG & ec00270 & Cysteine and methionine metabolism & 1.64E-02 & 1.64E-02 & 2 & 74 \\
\hline \multirow[t]{7}{*}{ TrypanoCyc } & SAM-PWY & S-adenosyl-L-methionine biosynthesis & $3.74 \mathrm{E}-04$ & $6.24 \mathrm{E}-05$ & 2 & 3 \\
\hline & PWY-802 & methionine and S-adenosylmethionine synthesis & $3.74 \mathrm{E}-04$ & 9.35E-05 & 2 & 4 \\
\hline & PWY0-781 & aspartate superpathway & $5.96 \mathrm{E}-04$ & 2.24E-04 & 2 & 7 \\
\hline & PWY1V8-2 & Methionine salvage cycle & 8.17E-04 & 4.09E-04 & 2 & 10 \\
\hline & PWY1V8-6 & Trypanothine biosynthesis and peroxide metabolism & $1.04 \mathrm{E}-03$ & $6.48 \mathrm{E}-04$ & 2 & 13 \\
\hline & METHIONINE-DEG1-PWY & methionine degradation I (to homocysteine) & $1.05 \mathrm{E}-02$ & 7.84E-03 & 2 & 50 \\
\hline & PWY0-381-1 & glycerol degradation I & 1.27E-02 & $1.11 \mathrm{E}-02$ & 1 & 3 \\
\hline
\end{tabular}

${ }^{a}$ Genes in the top 99-percentile RPKM dataset

${ }^{\mathrm{b}}$ Entire trypanosome genes in TritrypDB database in respective metabolic pathway

\section{Differential expression of $T$. congolense genes and enrichment analysis}

To characterize potential molecular differences between trypanosome developmental stages in cardia and proboscis, we analyzed DE genes between $\mathrm{C}$ - and $\mathrm{PB}$-parasite libraries. This comparison identified 2131 (15.95\%) DE genes, of which $59.17 \%$ exhibited significantly higher expression in C-parasites relative to PB-parasites (Fig. 1, Additional file 5: Table S2). Because of the limited number of biological replicates used, the $\mathrm{DE}$ genes that exhibit low FC (i.e. $\leq 2$ ) requires verification. The DE genes with the greatest fold change in favor of $\mathrm{C}$-parasites encoded receptor-type adenylate cyclases, transporter proteins (pteridine, amino acid and ABCs), putative cell-surface protein, procyclin-like protein, protein associated with differentiation 5 (PAD5) and zinc finger $\mathrm{ZC} 3 \mathrm{H} 22$ protein. Similarly, VSG, two invariant surface glycoproteins, three histones (two-H2B and $\mathrm{H} 4$ ), zinc finger type $\mathrm{C} 2 \mathrm{H} 2$ proteins and a number of hypothetical proteins were among those with the greatest fold change in $\mathrm{PB}$ compared to C-parasites (Fig. 1, Additional file 5: Table S2). RT-qPCR validation of our RNA-seq data revealed significant correlation (Pearson correlation = 0.9915) between these two analytical methodologies (Additional file 2: Text S2). 


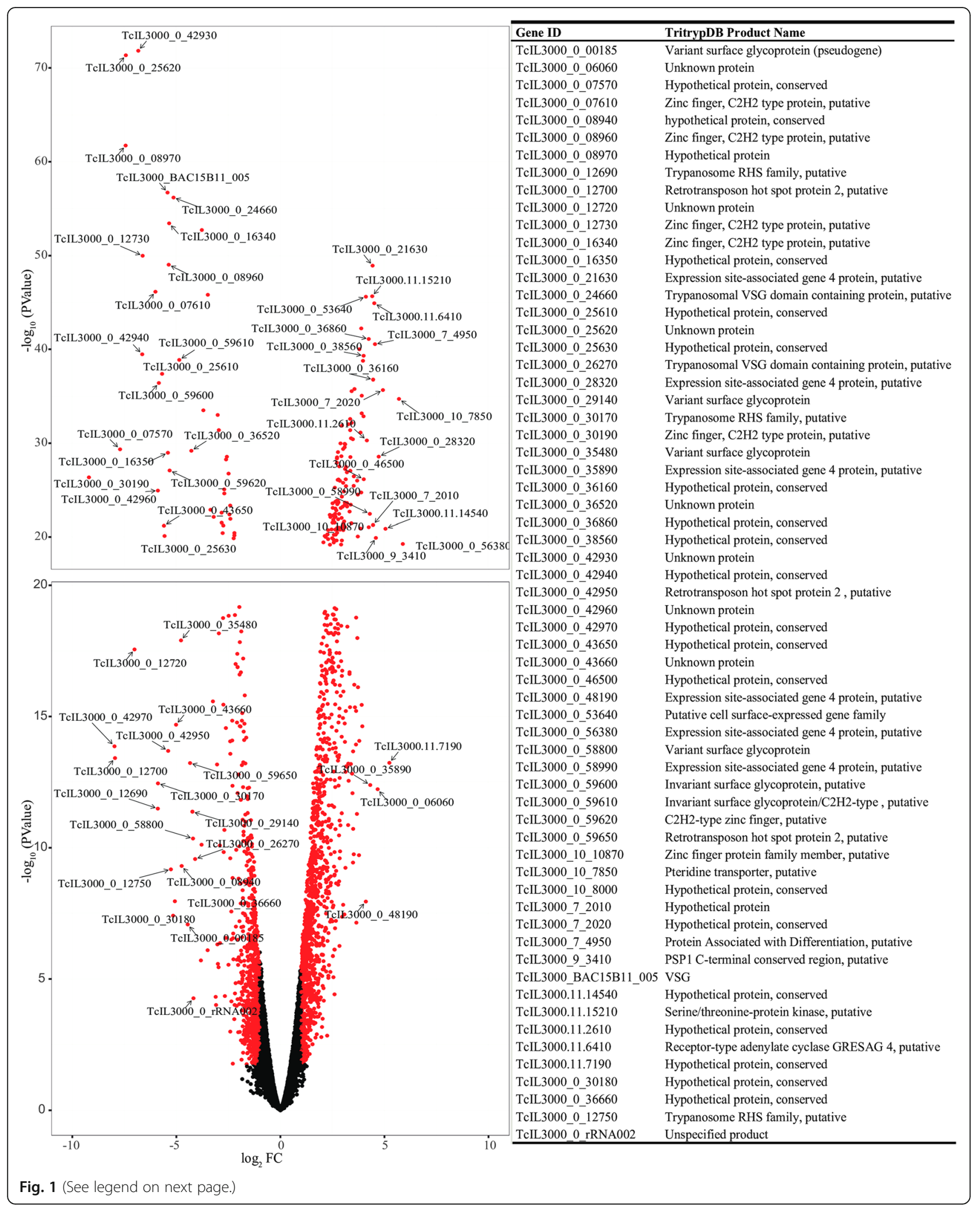


(See figure on previous page.)

Fig. 1 A Volcano plot showing differentially expressed genes of $T$. congolense parasites in the cardia relative to those in the probosces of G. morsitans. Only transcripts with at least 30 reads mapped and at least five RPKM (Reads Per Kilobase Million) by CLC-Genomics and EdgeR analysis [36, 99] in either library (C-parasites or PB-parasites) were considered. Red dots indicate DE genes with an FC of $\geq 2$ ( $\left.\log _{2}=1\right)$ and false detection rate (FDR) corrected $P$-value of $<0.05$ between cardia and proboscis parasites. The $x$-axis displays magnitude of fold-changes and $y$-axis the statistical significance ( $\log _{10}$ of $P$-value). Points having FC of $<2\left(\log _{2}<1\right)$ on an FDR corrected $P$-value of $<0.05$ are shown in black, and indicate genes with non-significance change between different developmental states

Blast2GO Fishers exact test of DE genes revealed 37 and five enriched Gene Ontology (GO) terms associated with $\mathrm{C}$ - and $\mathrm{PB}$-parasites, respectively (Additional file 6: Table S3). The GO enriched terms of putative proteins upregulated in C-parasites were broadly associated with nucleotides (nucleotide binding and cyclic nucleotide biosynthetic process; the energy carriers), ATP binding, protein phosphorylation, signaling, cell (integral component of cell membrane, membrane region, cytoplasmic side of plasma membrane), adenylate cyclase activity, quorum sensing as well as microtubules, protein serine/ threonine and transport processes. This suggests that $\mathrm{C}$-parasites are energetically active and can monitor and respond to their surroundings. Induction of several transport pathways in C-parasites suggests that these trypanosomes may be scavenging for nutrients in the cardia. The putative products of genes upregulated in the PB-parasite were associated with nucleosome, cytoplasmic part, membrane bound organelle, protein folding and protein heterodimerization activity; terms possibly reflecting high rate of cell division by parasites residing in this organ.

\section{Metabolic pathways}

African trypanosomes live in a glucose-rich environment in the mammalian hosts and in a glucose deficient environment in the tsetse vector. These contrasting habitats require trypanosomes to adjust their metabolic processes for energy production. Specifically, for energy production, $T$. congolense BSF parasites rely entirely on glycolysis and substrate level phosphorylation whereas midgut PCF parasites utilize oxidative phosphorylation. The KEGG [48] and TrypanoCyt [50] analyses of putative products of DE genes for enriched metabolic pathways identified pathways broadly linked with protein/amino acid metabolism (Fig. 2a). A survey of individual genes identified those that encoded proteins putatively associated with the Krebs cycle, respiratory chain and oxidative phosphorylation to be DE between $\mathrm{C}$-parasites and PB-parasites (Fig. 2b), suggesting an enhanced respiratory activity of $T$. congolense stages in both tsetse tissues. Trypanosome respiratory activity, coupled with the reactive oxygen intermediates produced by trypanosome infected tsetse tissues $[57,58]$, can result in the production of reactive oxygen species that leads to oxidative stress to both tsetse fly and trypanosomes. As such, we investigated if parasites in these two tissues produce antioxidants and NADPH that may protect them from oxidative damage. In this regard, we identified putative proteins that likely function as antioxidants (Fig. 2c) and in the pentose phosphate pathway (Fig. 2d), of which some were upregulated in $\mathrm{C}$-parasite and others in PB-parasites. Pentose phosphate pathway is an essential maintenance pathway that leads to generation of reducing agents in the form of NADPH (which act as an electron donor in detoxification reaction). The cells may use this pathway not only to protect themselves against oxidative stress, but also to produce ribose phosphates that function in nucleotide synthesis.

Putative proteins linked to lipid metabolism involving sterol biosynthesis and ether lipid metabolism were upregulated in $\mathrm{C}$ - and $\mathrm{PB}$-parasites, respectively (Fig. 2e). Increased expression of sterol synthesis genes and desaturase by $\mathrm{C}$-parasites may enable the generation of polyunsaturated fatty acids that may help to maintain parasite membrane fluidity under variable temperature conditions in the insect host as they move to the $\mathrm{PB}$ where they start to divide. Induced expression of lipid metabolism genes in PB-parasites suggest an increased activity of this pathway, probably indicating high lipid utilization for energy. Lastly, gene products that function in the glycolytic pathway were also DE (Fig. 2f). The majority of them, notably hexokinase, phosphofructokinase, fructose-bisphosphate aldolase and enolase, were upregulated in PB-parasites. The enhanced expression of hexokinase by the PB-parasites may be an indication of pre-adaptation of these parasites for the glucose-rich bloodstream environment in their mammalian host.

\section{Expression of genes regulating trypanosome differentiation} Trypanosoma congolense differentiates into several forms during development in tsetse before becoming mammalian infective MCFs in the PB. In T. brucei, this process is regulated by a number of proteins, including RNA-binding proteins (RBPs) [59], lipid phosphate phosphatases (LPP) [60] and/or Proteins Associated with Differentiation (PADs), also known as Major Facilitator Superfamily (MFS) transporters which are expressed in the mammalian stage of trypanosomes [61]. We identified 44 RBPs, five PADs and four LPPs that were DE (Fig. 3, Additional file 7: Table S4). Most of these RBPs were highly expressed in the C-parasites relative to PB-parasites. Even though most of the RBPs, especially in 


\begin{tabular}{|c|c|c|c|c|c|}
\hline \multicolumn{6}{|c|}{ Enriched metabolic pathways } \\
\hline \multirow[b]{2}{*}{ Tool } & \multirow[b]{2}{*}{ Pathway ID } & \multicolumn{4}{|l|}{ Higher in C-parasites } \\
\hline & & Pathway Description & Benjamini & Test" & Ref $^{* \%}$ \\
\hline \multirow[t]{6}{*}{ KEGG } & $\mathrm{ec} 00230$ & Purine metabolism & $2.79 \mathrm{E}-11$ & 55 & 191 \\
\hline & $\mathrm{ec} 00300$ & Lysine biosynthesis & 0.003179369 & 12 & 36 \\
\hline & ec00680 & Methane metabolism & 0.004520116 & 19 & 85 \\
\hline & ec00900 & Terpenoid backbone biosynthesis & 0.004520116 & 23 & 116 \\
\hline & ec00380 & Tryptophan metabolism & 0.0101047 & 19 & 97 \\
\hline & ec00562 & Inositol phosphate metabolism & 0.042242596 & 7 & 28 \\
\hline \multirow{4}{*}{ TrypanoCyc } & PWY4FS-6-1 & Phosphatidylethanolamine biosynthesis II & 0.042242596 & 4 & 11 \\
\hline & GLUCAT-PWY & Glutamate degradation IV & 0.042242596 & 3 & 6 \\
\hline & PWY1V8-10 & Erythroascorbate biosynthesis & 0.042242596 & 2 & 2 \\
\hline & & \multicolumn{4}{|l|}{ Higher in PB-parasites } \\
\hline Tool & Pathway ID & Pathway Description & Benjamini & Test* & $\operatorname{Ref}^{* *}$ \\
\hline \multirow[t]{17}{*}{ KEGG } & ec00020 & Citrate cycle (TCA cycle) & 0.048187409 & 4 & 32 \\
\hline & ec00030 & Pentose phosphate pathway & 0.042291692 & 4 & 21 \\
\hline & ec00051 & Fructose and mannose metabolism & 0.045083368 & 7 & 75 \\
\hline & ec00052 & Galactose metabolism & 0.045083368 & 2 & 6 \\
\hline & ec00253 & Tetracycline biosynthesis & 0.042291692 & 7 & 71 \\
\hline & ec00270 & Cysteine and methionine metabolism & 0.002292658 & 12 & 74 \\
\hline & ec00350 & Tyrosine metabolism & 0.042291692 & 7 & 64 \\
\hline & ec00400 & Phenylalanine, tyrosine and tryptophan biosynthesis & 0.042291692 & 3 & 12 \\
\hline & ec00480 & Glutathione metabolism & 0.042291692 & 5 & 31 \\
\hline & $\mathrm{ec} 00565$ & Ether lipid metabolism & 0.042291692 & 5 & 36 \\
\hline & ec00620 & Pyruvate metabolism & 0.048187409 & 4 & 32 \\
\hline & ec00941 & Flavonoid biosynthesis & 0.042291692 & 8 & 86 \\
\hline & ec00942 & Anthocyanin biosynthesis & 0.042291692 & 9 & 97 \\
\hline & ec00945 & Stilbenoid, diarylheptanoid and gingerol biosynthesis & is 0.042291692 & 8 & 88 \\
\hline & ec00950 & Isoquinoline alkaloid biosynthesis & 0.045973804 & 7 & 76 \\
\hline & ec01051 & Biosynthesis of ansamycins & 0.042291692 & 8 & 85 \\
\hline & ec01057 & Biosynthesis of type II polyketide products & 0.042291692 & 10 & 106 \\
\hline \multirow{15}{*}{ TrypanoCyc } & GLUTOXA-PWY & Glutamate degradation VII & 0.042291692 & 3 & 13 \\
\hline & $\begin{array}{l}\text { GLYCOLYSIS-TCA- } \\
\text { GLYOX-BYPASS }\end{array}$ & Superpathway of glycolysis, pyruvate dehydrogenase & 0.048187409 & 4 & 32 \\
\hline & LCTACACAT-PWY & $\begin{array}{l}\text { Lactate oxidation } \\
\text { Lactions }\end{array}$ & 0.042291692 & 2 & 4 \\
\hline & $\begin{array}{l}\text { MALATE-ASPARTATE- } \\
\text { SHUTTLE-PWY }\end{array}$ & Aspartate degradation II & 0.045083368 & 2 & 6 \\
\hline & $\begin{array}{l}\text { MANNOSYL-CHITO-DOL- } \\
\text { ICHOL-BIOSYNTHESIS }\end{array}$ & Dolichyl-diphosphooligosaccharide biosynthesis & 0.048187409 & 5 & 47 \\
\hline & METHIONINE-DEG1-PWY & Methionine degradation I (to homocysteine) & 0.042291692 & 7 & 50 \\
\hline & PWY-7219 & Adenosine ribonucleotides-de novo-biosynthesis & 0.042291692 & 4 & 27 \\
\hline & PWY-802 & Methionine and S-adenosylmethionine synthesis & 0.042291692 & 2 & 4 \\
\hline & PWY0-781 & Aspartate superpathway & 0.042291692 & 3 & 7 \\
\hline & PWY1V8-2 & Methionine salvage cycle & 0.002719749 & 5 & 10 \\
\hline & PWY1V8-6 & Trypanothine biosynthesis and peroxide metabolism & 0.042291692 & 3 & 13 \\
\hline & PWY1V8-8 & Acetate/acetyl-CoA metabolism & 0.042291692 & 4 & 25 \\
\hline & PWY1V8-9 & Succinate and Acetate/Acetyl CoA metabolism & 0.042291692 & 5 & 41 \\
\hline & SAM-PWY & $\mathrm{S}$-adenosyl-L-methionine biosynthesis & 0.042291692 & 2 & 3 \\
\hline & VALDEG-PWY & Valine degradation I & 0.048187409 & 2 & 7 \\
\hline
\end{tabular}

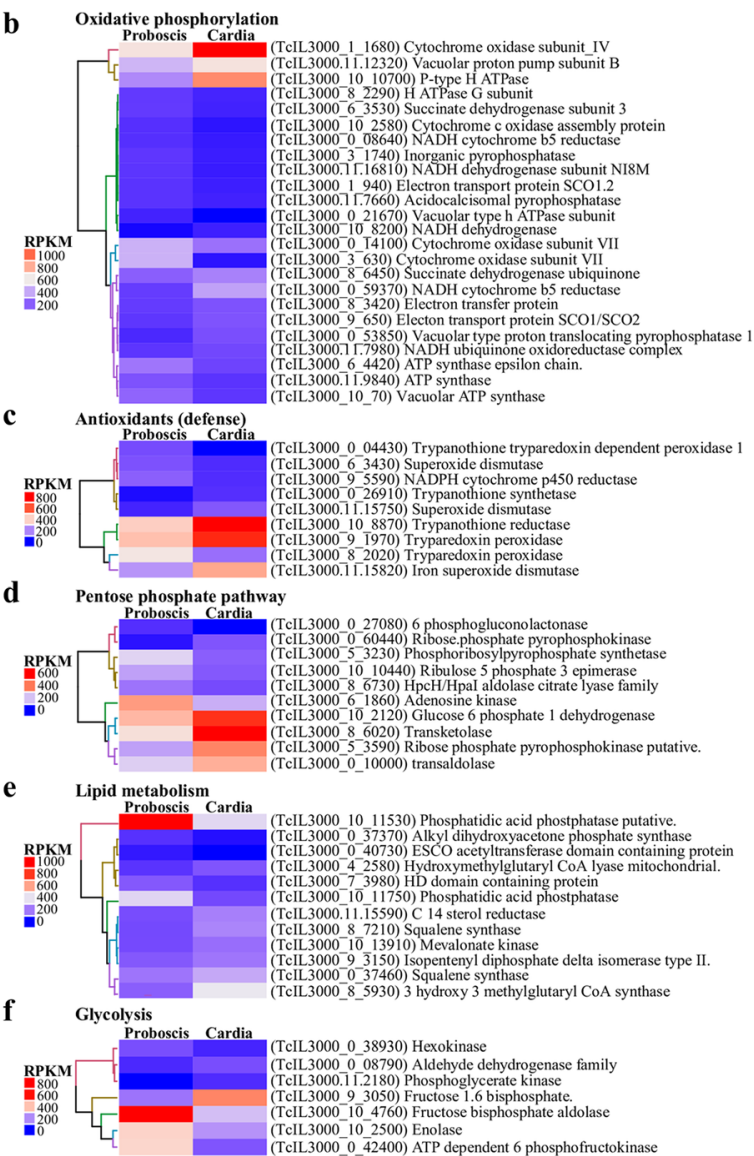

Fig. 2 Differential expression of genes in metabolic pathways. Heat maps showing the expression profiles consisting of respective RPKMs clustered using Euclidean distance calculation and ward.D clustering methods. a KEGG and TrypanoCYC enriched metabolic pathway. b-f Genes that function in the oxidative phosphorylation pathway, antioxidant (defense), pentose phosphate pathway, lipid metabolism, and glycolytic pathway respectively

T. congolense, are not functionally annotated, it is worth noting that ZC3H20 and RBP6 were abundant in C-parasites. In T. brucei, $\mathrm{ZC} 3 \mathrm{H} 20$ is required for growth of PCF, while RBP6 is involved in differentiation to MCF [62, 63]. The RBPs that exhibited higher expression in PB-parasites relative to C-parasites included ALBA3, RBSR2,3, RBP7A and Zinc finger protein (ZC3H24). ALBA3 in T. brucei is expressed by all parasite stages except for those in the cardia, and artificial overexpression of this protein disturbs normal trypanosome development process taking place in the cardia [64]. All DE PADs were highly expressed in C-parasites compared to PB-parasites, with the exception of only two that were upregulated in PB-parasites. In contrast to RBPs and PADs, the expression of all LPPs was higher in PB-parasites relative to C-parasites, mirroring results observed in T. brucei in tsetse's SG [60].

\section{Differential expression of $T$. congolense cell-surface proteins (CSPs)}

The surface of $T$. congolense is covered by a dense layer of glycoprotein, the composition of which is characteristic of each differentiation stage. Most of these glycoproteins are attached to the plasma membrane by a glycosylphosphatidyl inositol (GPI) anchor or are transmembrane (TM), and have been grouped into multigene families [42]. Of the DE gene dataset, 223 encoded CSPs based on the presence of putative GPI-anchor or TM protein domains (Fig. 4, Additional file 8: Table S5). For the putative GPI-anchored proteins (Fig. 4a), our analyses show that all the six genes putatively encoding 'GARP' and 'iii' subfamily proteins, and the single protein of ' $\mathrm{i}$ ' subfamily of the Fam50 [42], were upregulated in C-parasites. In addition, two Fam12, four Fam47, three Fam51 and several hypothetical GPI-anchored protein coding genes were also induced in C-parasites. The GPI-anchored protein-encoding genes induced in the PB-parasites included ten putative VSG proteins, of which three were metacyclic-specific VSGs [m-VSG-3, 6, 10; (Fig. 4b)]. In the C-parasite dataset, one upregulated gene encoded a putative VSG (TcIL3000_0_07340). Apart from the VSGs, other upregulated genes in PB-parasites that encoded putative GPI-anchored CSPs included Fam50's three 'CESP' 


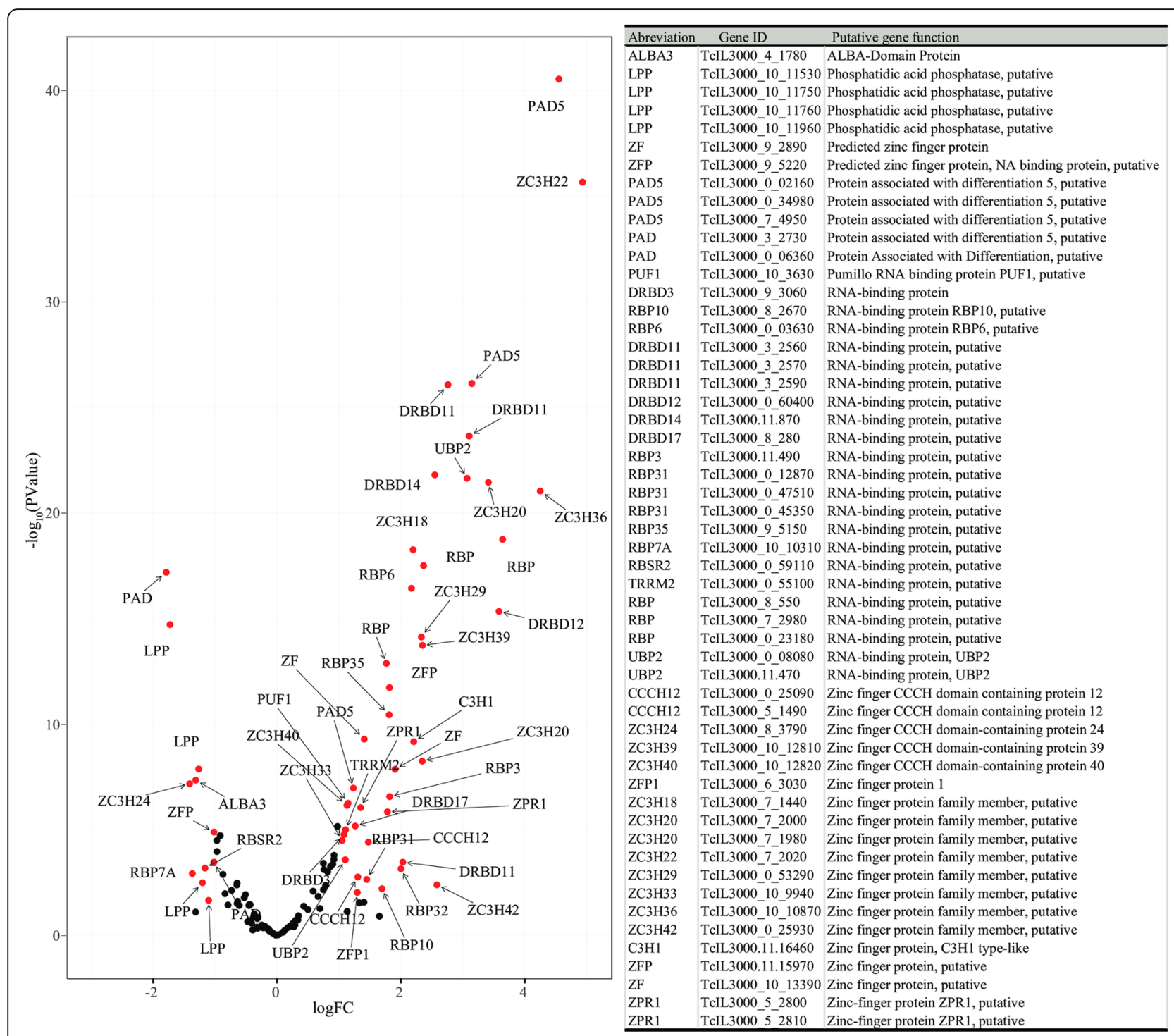

Fig. 3 Volcano plot showing DE genes between cardia and proboscis parasites encoding proteins putatively associated with trypanosome differentiation. The red dots indicate points-of-interest with a fold-change of $\geq 2\left(\log _{2}=1\right)$ and False Detection Rate (FDR) corrected $P<0.05$ between cardia and proboscis parasites. Black dots represent genes with no significance change in expression level

subfamily genes, the single (TcIL3000_0_02370) subfamily 'iv' gene as well as two Fam46 (major surface protease gp63) and Fam14 (procyclin-associated gene; PAG1-2,4-5) [42]. Also, upregulated in PB-parasites were genes encoding a haptoglobin-hemoglobin receptor protein, an amastin surface glycoprotein and several hypothetical proteins.

For the genes encoding putative TM proteins, we found 14 members of Fam51, several members of transporter families (Fam54, 56, 57, 59, 60, 61, 62), a lipase (Fam63) and a zinc-finger (Fam76) to be highly expressed by the C-parasites compared to PB-parasites (Fig. 4b, Additional file 8: Table S5). On the other hand, in the PB-parasites, at least a member of different gene families was induced relative to the C-parasite (Fig. $4 \mathrm{~b}$,
Additional file 8: Table S5). Of these gene families, Fam67 (cysteine proteases) attracted considerable interest [65], more so in T. congolense [66], where they are thought to have a direct impact on disease pathogenesis as virulence factors involved in host invasion, migration, metabolism and immune evasion. Expression of cysteine protease encoding genes by the insect stage parasites mirrors results found in previous proteome [26] and transcriptome [25] studies in the insect stages, suggesting that these proteins may also play important roles vector-parasite interactions. Importantly, genes highly expressed by PB-parasites are likely to encode proteins that are transmitted to mammalian hosts, and this may assist the parasite in overcoming the many defense 
a

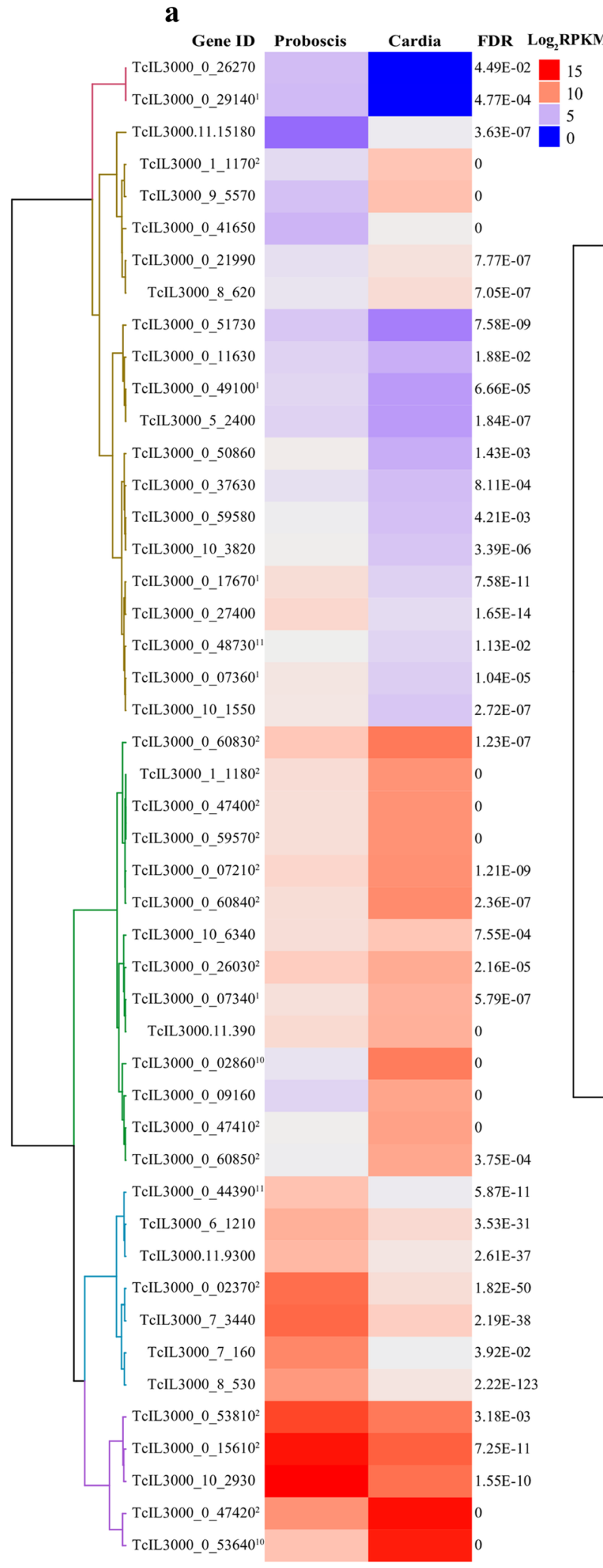

b

\begin{tabular}{|c|c|c|c|}
\hline Gene ID & Proboscis & Cardia & $\log _{2}$ RPKM \\
\hline TcIL3000_0_06950 & & & $9.68 \mathrm{E}-03$ \\
\hline TcIL3000_0_567306 & & & $2.74 \mathrm{E}-13-12$ \\
\hline TcIL3000_0_14520 & & & $1.91 \mathrm{E}-13$ \\
\hline TcIL3000.11.6750 & & & $5.55 \mathrm{E}-08$ \\
\hline TcIL3000_9_2160 & & & $1.14 \mathrm{E}-03$ \\
\hline TcIL3000_0_26130 & & & $1.39 \mathrm{E}-06$ \\
\hline TcIL3000_10_7320 & & & 0 \\
\hline TcIL3000_10_7850 & & & $3.69 \mathrm{E}-06$ \\
\hline TcIL3000_0_45250 & & & 0 \\
\hline TcIL3000_0_19060 & & & 0 \\
\hline TcIL3000_0_48390 & & & $1.04 \mathrm{E}-03$ \\
\hline TcIL3000_0_498804 & & & 0 \\
\hline TcIL3000_10_6590 & & & 0 \\
\hline TcIL3000_0_139704 & & & $2.26 \mathrm{E}-04$ \\
\hline TcIL3000_0_483804 & & & 4.79E-04 \\
\hline TcIL3000_0_569907 & & & 0 \\
\hline TcIL3000_0_30940 & & & $8.54 \mathrm{E}-06$ \\
\hline TcIL3000_7_6140 & & & 3.01E-09 \\
\hline TcIL3000_0_13930 & & & $1.43 \mathrm{E}-02$ \\
\hline TcIL3000.11.6640 & & & $1.48 \mathrm{E}-10$ \\
\hline TcIL3000_10_2060 & & & 0 \\
\hline TcIL3000_0_001204 & & & 0 \\
\hline TcIL3000_0_16450 3 & & & 0 \\
\hline TcIL3000_0_02830 & & & 0 \\
\hline TcIL3000_0_23420 3 & & & 0 \\
\hline TcIL3000_0_60800 3 & & & 0 \\
\hline TcIL3000_0_45440 3 & & & $1.99 \mathrm{E}-06$ \\
\hline TcIL3000_0_484304 & & & $1.85 \mathrm{E}-02$ \\
\hline TcIL3000_0_13340 & & & $2.98 \mathrm{E}-08$ \\
\hline TcIL3000_0_55070 & & & $6.85 \mathrm{E}-05$ \\
\hline TcIL3000_0_139504 & & & 0 \\
\hline TcIL3000_0_48400 & & & $2.03 \mathrm{E}-14$ \\
\hline TcIL3000_0_53850 & & & $5.83 \mathrm{E}-05$ \\
\hline TcIL3000.11.3590 & & & $2.61 \mathrm{E}-02$ \\
\hline TcIL3000_0_42720 & & & $1.93 \mathrm{E}-02$ \\
\hline TcIL3000_8_8240 & & & $3.48 \mathrm{E}-03$ \\
\hline TcIL3000.11.6410 & & & $7.81 \mathrm{E}-10$ \\
\hline TcIL3000_0_29870 & & & 0 \\
\hline TcIL3000_5_23207 & & & $2.06 \mathrm{E}-10$ \\
\hline TcIL3000_0_05730 & & & 0 \\
\hline TcIL3000_0_09250 & & & $2.24 \mathrm{E}-03$ \\
\hline TcIL3000_0_35890 & & & $1.13 \mathrm{E}-02$ \\
\hline TcIL3000_0_17840 & & & 4.99E-09 \\
\hline TcIL3000_0_34290 & & & $2.74 \mathrm{E}-05$ \\
\hline TcIL3000_0_07220 3 & & & 0 \\
\hline TcIL3000_0_00660 & & & $7.05 \mathrm{E}-03$ \\
\hline TcIL3000_0_19690 & & & $1.34 \mathrm{E}-02$ \\
\hline TcIL3000_0_24060 & & & $8.31 \mathrm{E}-03$ \\
\hline TcIL3000_10_14040 & & & $3.05 \mathrm{E}-09$ \\
\hline TcIL3000_6_609 & & & $2.75 \mathrm{E}-27$ \\
\hline TcIL3000_3_960 & & & $5.17 \mathrm{E}-10$ \\
\hline TcIL3000_0_03600 & & & $6.82 \mathrm{E}-55$ \\
\hline TcIL3000.11.13590 & & & $2.67 \mathrm{E}-02$ \\
\hline TcIL3000_0_33420 & & & $4.28 \mathrm{E}-02$ \\
\hline TcIL3000_6_330 & & & $2.25 \mathrm{E}-02$ \\
\hline TcIL3000_8_3800 & & & $6.75 \mathrm{E}-20$ \\
\hline TcIL3000_0_18160 & & & $7.12 \mathrm{E}-03$ \\
\hline TcIL3000.11.9350 & & & $5.24 \mathrm{E}-04$ \\
\hline TcIL3000_0_03060"1 & & & 3.48E-10 \\
\hline TcIL3000_0_06720 & & & $8.24 \mathrm{E}-24$ \\
\hline TcIL3000_0_45330 & & & $5.68 \mathrm{E}-08$ \\
\hline TcIL3000_0_56420 & & & $7.90 \mathrm{E}-08$ \\
\hline TcIL3000_6_320 & & & $2.87 \mathrm{E}-08$ \\
\hline TcIL3000_0_28850"1 & & & $5.49 \mathrm{E}-08$ \\
\hline TcIL3000.11.3570 & & & $3.41 \mathrm{E}-03$ \\
\hline TcIL3000.11.8970 & & & $2.77 \mathrm{E}-02$ \\
\hline TcIL3000_10_8520 & & & $3.77 \mathrm{E}-04$ \\
\hline TcIL3000_9_2500 & & & $1.09 \mathrm{E}-02$ \\
\hline
\end{tabular}

Fig. 4 (See legend on next page.) 
(See figure on previous page.)

Fig. 4 Heatmaps of expression of T. congolense genes encoding putative cell surface proteins. a GPI anchored proteins. Prediction of the putative GPI-anchored cell surface proteins was determined by FragAnchor [44], PredGPI [43] and BigPI [45]. b Transmembrane proteins. Trans-membrane helices was predicted using TMHMM [46]. The expression profiles consist of respective $\log _{2}$ transformed RPKM clustered using Euclidean distance calculation and ward.D clustering methods. ${ }^{1}$ Fam13/16 (VSGs), ${ }^{2}$ Fam50 (Brucei alanine-rich protein), ${ }^{3}$ Fam51 (Expression site-associated gene 4),

${ }^{4}$ Fam54 (Amino acid transporter), ${ }^{5}$ Fam56 (ABC transporter), ${ }^{6}$ Fam57 (Folate transporter, ESAG10), ${ }^{7}$ Fam60 (Membrane transporter protein), ${ }^{8}$ Fam61 (Nucleoside transporter), ${ }^{9}$ Fam67 (Cysteine peptidase), ${ }^{10}$ Fam12 (Procyclin-like), ${ }^{11}$ Fam14 (Transferrin receptor-like, PAG-like)

factors produced by the host immediately upon their entry in the animal.

The T. congolense genome also encodes parasite species specific CSPs. These T. congolense unique CSPs (grouped into Fam17-22) may help distinguish this parasite from T. brucei and T. vivax [42]. Analysis of these genes revealed that they exhibited relatively low expression levels in both C- and PB-parasites (Additional file 9: Figure S2). Nonetheless, some members of Fam17 and Fam18 were upregulated in the $\mathrm{C}$-parasites relative to PB-parasites, while three members of Fam 20 and a member of Fam21 showed higher expression in PB-parasites relative to $\mathrm{C}$-parasites.

\section{Developmental stage-regulated expression of selected CSPs}

Semi-quantitative RT-PCR analysis was used to track the tissue and developmental stage-regulated expression of selected CSP encoding genes following normalization of samples using $T$. congolense gapdh across all tsetse tissues (midgut, cardia and proboscis) and BSF (Fig. 5a). Genes encoding GPI-anchor GARP, putative cell surface protein (TcIL3000_11_47420), and TM Receptor adenylate cyclase (GRESAG4, TcIL3000.11.6410) were most abundant in the cardia relative to other tsetse tissues and BSF parasites. Similarly, expression of TM Pteridine transporter (TcIL3000_10_7850) was higher in parasites colonizing the cardia and midgut than in the PB and BSF. The GPI-anchored Haptoglobin-hemoglobin (TcIL3000_10_ 2930), CESP family and hypothetical protein genes (TcIL3000_0_02370 and TcIL3000_7_3440) showed higher expression in PB-parasites than those colonizing other tissues (Fig. 5b).

The products of the two hypothetical genes (TcIL3000_0_02370 and TcIL3000_7_3440) have GPIanchor motifs, and also showed higher expression in $\mathrm{PB}$-parasites relative to C-parasites in both semi-quantitative RT-PCR and RNA-seq analysis. Hence the expression profiles of these genes were further tested in different infected fly tissues and BSF parasites by RT-qPCR. The ANOVA analysis of RT-qPCR results of these two genes indicate that they were both significantly $\left(F_{(3,28)}=21.39, P<0.0001\right)$ highly expressed by $\mathrm{PB}$-parasites relative to parasites rest of the tsetse tissues and BSF (Fig. 5c).

We next expressed the TcIL3000_0_02370 protein in bacteria and generated rabbit polyclonal antibodies against the recombinant protein. These antibodies were then used to localize TcIL3000_0_02370 protein in parasites derived from distinct tsetse tissues. The hypothetical protein (TcIL3000_0_02370) which is predicted to be glycosylated, contain 453-amino acids and encoded a putative GPI-anchor (position 429) and signal peptide motif (positions 1-22). This putative protein was chosen for IFA analysis because its orthologue in T. brucei is expressed preferentially by metacyclic stage parasites [52]. Based on immunofluorescent and confocal laser microscopy analysis, the expression of TcIL3000 0_02370 protein was found to be localized on the surface of parasites residing in the $\mathrm{PB}$, with minor staining also observed in C-parasites (Fig. 5d). The minor staining in the $\mathrm{C}$-parasites may indicate that the expression of this protein probably starts in the population of parasites residing in the cardia and reaches its peak expression when parasites differentiate to EMF in the $\mathrm{PB}$, as previously described in proteomic analysis of $T$. congolense four life-cycle stages [26].

\section{Discussion}

In this study, we used high throughput RNA expression analysis to examine molecular differences of $T$. congolense developmental forms colonizing the cardia (C-parasites) and proboscis (PB-parasites) of G. m. morsitans. Our results reveal that $\mathrm{C}$-parasites express genes that encode products linked with nucleotides/nucleosides, cell signaling and quorum sensing (QS), and several transport systems, suggesting parasite adaptation to varying nutritional environments in the vector. PB-parasites, on the other hand, express putative proteins associated with cell proliferation, in line with previous finding [12]. Greater cell division processes observed in PB-parasites may either enable multiplication of EMF or generation of short MCFs of $T$. congolense from long EMF [12]. In cultures of long attached EMFs, MCFs only appeared if short, attached trypomastigotes were present [67].

As extracellular parasites, African trypanosomes, including $T$. congolense, sense and respond appropriately to changes in their host environment. This feature is critical for the parasite to monitor nutrient availability, space and prevent accumulation of toxic metabolic waste for survival [68]. This process likely involves a repertoire of receptors, reporter molecule(s) and signaling pathways, 


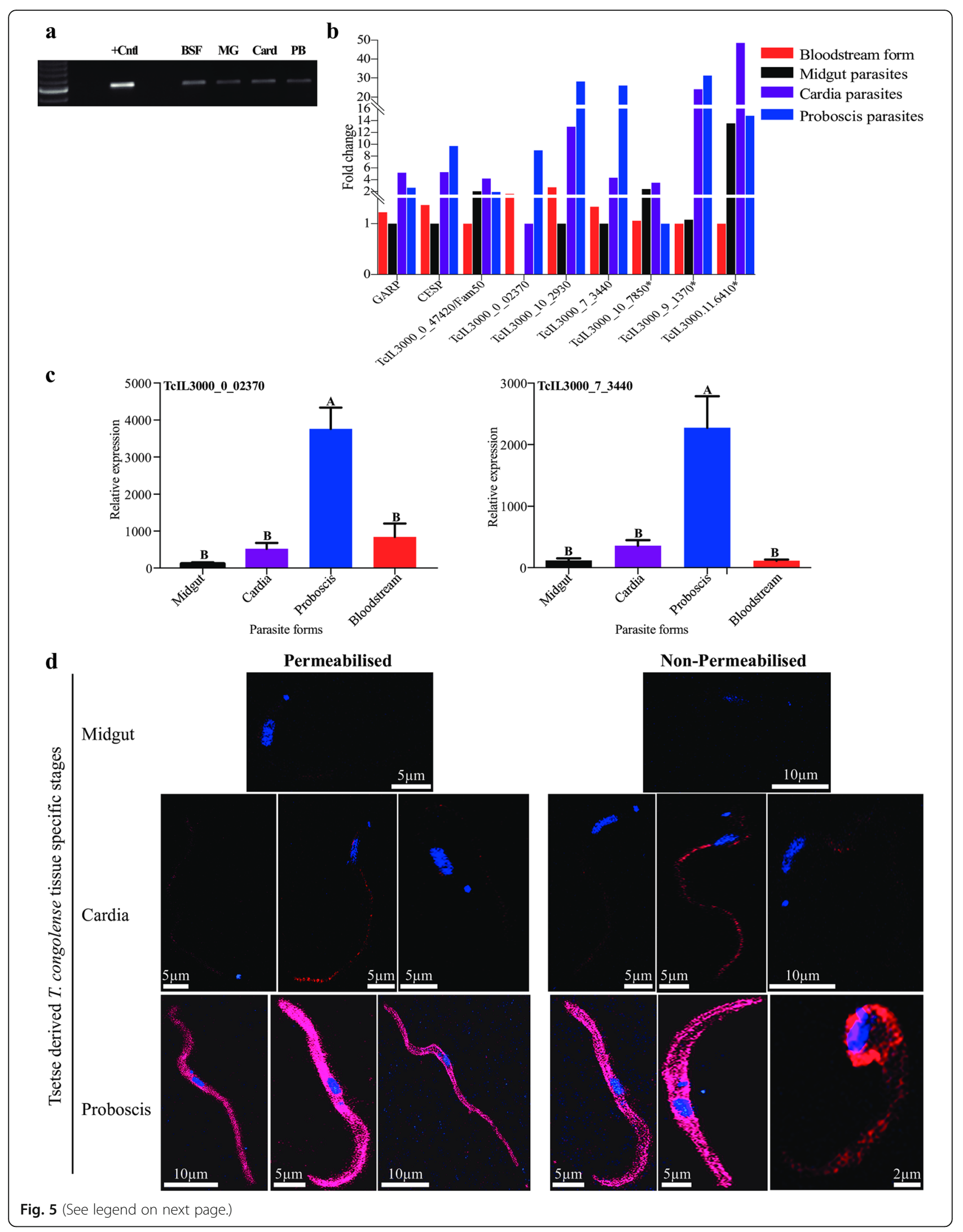


(See figure on previous page.)

Fig. 5 Expression analysis of selected CSP genes. a Expression levels of T. congolense gapdh from infected tsetse's midgut, cardia and proboscis organs as well as bloodstream form parasites purified from infected mice blood. Abbreviations: BSF, bloodstream form; MG, tsetse midgut parasites; card, cardia parasites; PB, proboscis parasites. b Stage-regulated gene expression profiles for genes that putatively encode GPI-anchored and transmembrane proteins, normalized to gapdh levels as shown in A. * indicates transmembrane protein encoding genes. $\mathbf{c}$ Expression analysis of two GPI-anchored protein encoding genes via RT-qPCR. $\mathbf{d}$ Localization of T. congolense TcIL3000_0_02370 protein in parasites residing in the midgut, cardia and proboscis organs examined by immunofluorescent staining and confocal laser microscopy. Red indicates immunofluorescence staining of TclL3000_ 0_02370, and blue indicates the DAPI staining of nucleus and kinetoplast

with some evidence that cAMP and QS may also play important roles in this cascade $[68,69]$. Increased expression of receptor-like adenylate cyclases (ACs; ESAG4/GRESSAG), QS and signal transduction proteins in C-parasites suggest that these individuals can also monitor their density [68] and engage in social behaviors as described for procyclic T. brucei [70-72]. The African trypanosome genomes encode an unusually expanded repertoire of ACs, with T. brucei encoding over 80 and T. congolense 45 [73]. This expansion only exists in the extracellular trypanosomes, but not in related intracellular kinetoplastids like T. cruzi and Leishmania spp. In T. brucei, one well studied member of subfamily of ACs is an expression site associated gene (ESAG4), which is a BSF stage specific flagellar pocket protein involved in disrupting host innate immunity [73]. Insect stage-specific T. brucei ACs have also been identified, suggesting similar roles during parasite development in the tsetse vector $[13,74]$. ACs can also control cell division [75] and signal for social motility [76]. As such, upregulation of T. congolense ACs in the cardia may be linked to increased function of cAMP mediated signaling pathways and social motility [69], as C-parasites do not divide [12]. Aspects of QS and signal transduction-like social activities, including social motility, can facilitate trypanosome movement in the tsetse vector [70], and has been postulated for T. brucei procyclics in tsetse midgut [77, 78]. QS may also boost host nutrient accessibility while overcoming host defenses for survival [72]. Our results suggest that $T$. congolense $\mathrm{C}$-parasites may also engage in social behaviors that can enable them to move from the cardia en masse to colonize the foregut as well as maximize the ability of these parasites to acquire nutrients from the cardia. The nutrient transporter proteins expressed by C-parasites may further enhance parasite uptake of metabolites in the cardia lumen.

We also detected expression of genes whose products potentially regulate parasite differentiation, e.g. RBPs, PADs and LPPs [59-61, 63, 79]. In T. brucei, overexpression of the gene encoding RNA Binding protein 6 (RBP6) using pLEW100 vector in in vitro cultured noninfectious PCF led to the generation of mammalian infective MCF [63]. The zinc finger protein, $\mathrm{ZC} 3 \mathrm{H} 20$, regulates differentiation and growth of PCF [79], while ZC3H11 stabilizes heat-shock protein 70 and enables survival of BSF in the animal host [80]. PADs have been identified in T. brucei, where PAD2 enables differentiation of BSF cells to procyclics during the early stages of fly midgut infection [61]. The expression of all LPPs were induced in T. congolense PB-parasites similar to their T. brucei homologs, which are also induced in tsetse's SG [60]. The increased expression of LPPs might be important for the uptake of phospholipids from PB by the attached parasites, in order to support parasite growth, proliferation and differentiation in the PB [81, 82].

Several African trypanosome CSPs that may be involved in host-parasite interactions have been identified [42]. Our analysis revealed that all members of Fam50 subfamily 'iii', ' $i$ ' and 'GARP' are induced in C-parasites while three members of the 'CESP' subfamily are induced in PB-parasites. This result contradicts previously held knowledge based on in vitro cultured $T$. congolense stages where GARP is preferentially expressed by EMF stage parasites $[26,83]$ in tsetse's $P B[12]$. While the roles of GARP and 'iii' subfamily of proteins are unknown, CESP protein is thought to enable EMF attachment to the PB wall [84]. The levels of four invariant surface glycoproteins (ISGs), the Haptoglobin-hemoglobin receptor $(\mathrm{HpHbR})$ and several VSGs, including three previously identified metacyclic specific-VSGs, are induced in PB-parasites [25, 26]. The VSGs may be a preadaptation strategy of the parasite for transmission to the animal host and evasion of host immunity [85]. The role of ISGs in $T$. congolense is unknown, but their $T$. brucei homolog (ISG75) functions in suramin metabolism in BSFs [86]. The $\mathrm{HpHbR}$ of $T$. congolense has high affinity for hemoglobin and is strategically expressed on the surface of EMF parasites $[26,87]$, which appear in the PB and cibarium. In the $\mathrm{PB}$, the $\mathrm{HpHbR}$ may enable the parasites to acquire heme from incoming blood during tsetse feeding $[87,88]$. In T. brucei, HpHbR is BSF-specific, where it helps the parasite to acquire haptoglobin-hemoglobin complexes for heme [89]. In addition, several hypothetical CSPs were also induced in PB-parasites, notably TcIL3000_0_02370, which is thought to be involved in EMF to MCF development [26]. This single copy gene (TcIL3000_0_02370), has multi-copy orthologues (Tb927.7.360) in T. brucei's genome and is preferentially expressed by SG colonizing parasites [52].

Given the small number of MCF parasites [90] that get deposited with saliva into the mammalian host bite 
site, the metacyclic developmental stage presents a bottleneck for transmission. If MCF-specific proteins can be effectively targeted at the bite site, it could block parasite infection establishment in animal hosts. Towards this end, studies in mice immunized with radiation-attenuated trypanosomes collected from animal blood 5-days post-challenge with infected tsetse showed protection against a subsequent parasite challenge. Again, mice that were challenged twice with $T$. congolense infected tsetse then treated with trypanocidal drugs after each challenge, and finally followed by homologous parasite challenge, resulted in sterile immunity [91, 92]. However, immunity to trypanosomes in these experiments was short-lived [93]. Such strategies have also been tried in Plasmodium spp. by targeting the CS antigen of sporozoites from mosquito SG [94, 95] and other antigens [96] to block transmission. Proteins expressed by immature parasite stages in the fly also present possible targets for genetic modification to prevent parasite maturation in the vector [97]. However, it should be noted that $T$. congolense populations, particularly those in tsetse's $\mathrm{PB}$, are variable, and gene expression analyses of these parasites is complex and difficult to tease apart.

\section{Conclusions}

Collectively, our results provide insight into $T$. congolense gene expression profiles in two distinct niches within the tsetse vector. Results from C-parasites suggest that $T$. congolense parasites in the cardia may exhibit social motility. In addition, we identified a number of genes encoding putative cell surface proteins and proteins associated with parasite differentiations that may regulate $T$. congolense developmental processes in tsetse fly. Genes encoding transporter proteins abundantly expressed by C-parasites may aid the parasite in acquiring nutrients from the cardia. Our findings together with the previous proteome and transcriptomic data based on in vitro cultured parasites $[25,26]$, provide resources to facilitate further research into the life-cycle of $T$. congolense in the tsetse vector and expand the toolbox for transmission control.

\section{Additional files}

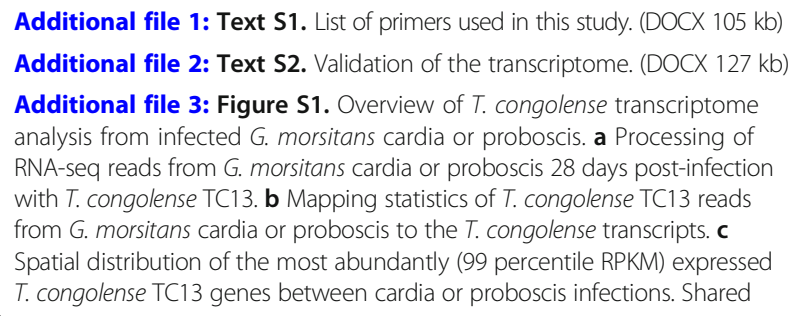
analysis from infected G. morsitans cardia or proboscis. a Processing of RNA-seq reads from $G$. morsitans cardia or proboscis 28 days post-infection with $T$. congolense TC13. b Mapping statistics of T. congolense TC13 reads from G. morsitans cardia or proboscis to the T. congolense transcripts. c Spatial distribution of the most abundantly (99 percentile RPKM) expressed T. congolense TC13 genes between cardia or proboscis infections. Shared

genes with higher expression in cardia or proboscis are indicated with '*' in respective directions. (TIF $1642 \mathrm{~kb}$ )

Additional file 4: Table S1. The top 99 percentile (RPKM) most highly expressed genes in cardia and proboscis. (XLSX $63 \mathrm{~kb}$ )

Additional file 5: Table S2. Differentially expressed genes between C- and PB-parasites. Sheet 1. Differently Expressed genes ( $F C \geq 2$ ) between C-parasites and PB-parasites with FDR, $P<0.05$. Sheet 2 . Moderately differently expressed genes $(F C<2)$ between $C$-parasites and PB-parasites with FDR, $P<0.05$. Sheet 3. Differently expressed genes ( $F C \geq 2$ ) between $C$-parasites and PB-parasites with FDR, $P<0.05$ only with CLC-Genomics tool. Sheet 4 . Differently expressed genes ( $F C \geq 2$ ) between C-parasites and PB-parasites with FDR, $P<0.05$ only with EdgaR software. Sheet 5. Differently expressed genes $(F C \geq 2)$ between C-parasites and PB-parasites with FDR, $P<0.05$ with proteomics data from [25]. (XLSX $1187 \mathrm{~kb})$

Additional file 6: Table S3. Gene ontology analysis of differentially expressed gene products between C-parasites and PB-parasites. (XLSX $31 \mathrm{~kb}$ )

Additional file 7: Table S4. Analysis of genes coding for proteins associated with differentiation. (XLSX $62 \mathrm{~kb}$ )

Additional file 8: Table S5. Expression analysis of $T$. congolense genes encoding cell surface proteins. (XLSX $90 \mathrm{~kb}$ )

Additional file 9: Figure S2. Heatmaps showing expression of $T$. congolense-specific genes belonging to Cell Surface Phylome families 17-21. The genes in these families were obtained [42] deposited in GeneDB [98]. The expression profiles consist of respective Log $_{2}$ transformed RPKM clustered using Euclidean distance calculation and ward.D clustering methods. (TIF $1937 \mathrm{~kb}$ )

\section{Abbreviations}

AAT: Animal African trypanosomosis; AC: Receptor-like adenylate cyclase; BCA: Bicinchoninic acid assay; BSF: Bloodstream form trypanosomes; CDNA: Complemetary deoxyribonucleic acid; CESP: Congolense ecpimastigote specific protein; CSP: Cell surface protein; DAPI: 4',6-diamidino-2-phenylindole; DE: Differentially expressed genes; EMF: Epimastigote form trypanosome; FDR: False discovery rate; GARP: Glutamic acid alanine rich protein; GO: Gene Ontology; GPI: Glycosylphosphatidyl inositol; HpHbR: Haptoglobin-hemoglobin receptor; IPTG: Isopropyl-ß-thiogalactoside; ISG: Invariant surface glycoprotein; KEGG: Kyoto Encyclopedia of Genes and Genomes; LPP: Lipid phosphate phosphatases; MCF: Metacyclic form trypanosomes; m-VSG: Metacyclic-specific variant surface glycoprotein; NCBI: Centre for Biotechnology Information; PAD: Proteins associated with differentiation; PB: Proboscis; PCF: Procyclic form trypanosome; PCR: Polymerase chain reaction; PSG: Phosphate buffered saline glucose; QS: Quorum sensing; RBP: RNA-binding protein; RPKM: Reads per kilobase of transcripts per million mapped reads; RT-qPCR: Real-time quantitation polymerase chain reaction; TM: Transmembrane; VAT: Variant antigenic type; VSG: Variant surface glycoprotein

\section{Acknowledgments}

We thank Dr Geoffrey Attardo for helpful discussions for RNA-seq analysis, Dr Xiaoli Bing for helping with tsetse dissection, Emre Aksoy for generating some of the RNA-seq libraries, members of the Aksoy lab at Yale University and Medical Biochemistry laboratory of Biotechnology Research Institute for technical assistance during this study.

\section{Funding}

This work was supported by National Institutes of Health (http://www.nih.gov) Fogarty Center grant D43TW007391, RO3TW009444, and National Institute of Allergy and Infectious Diseases grant RO1A1051584 awarded to SA.

\section{Availability of data and materials}

The data supporting the conclusions of this article are included within the article and its additional files. All sequences are available in the Sequence Read Archive number SRP093558 and PRJNA354110 for infected cardia and proboscis respectively, at the NCBI.

\section{Authors' contributions}

Conceptualization: EOA, POM, GM, SA. Sample collection: EOA, BLW, AV. Sample processing: EOA, BLW, AV, SA. Data generation: EOA, AV, SA. Data 
analysis: EOA, BLW, POM, AV, SA. Manuscript development: EOA, POM, BN. Manuscript review: BLW, AV, GM, SA. Supervision: BLW, POM, AV, BN, GM, SA. All authors read and approved the final manuscript.

\section{Ethics approval}

Animal experiments were carried out in strict accordance with recommendations in the Office of Laboratory Animal Welfare at the National Institutes of Health and Yale University Institutional Animal Care and Use Committee (ACUC). The experimental protocol was reviewed and approved by the Yale University Institutional Animal Care and Use Committee (Protocol number 2014-07266).

\section{Consent for publication}

Not applicable

\section{Competing interests}

The authors declared that they have no competing interests.

\section{Publisher's Note}

Springer Nature remains neutral with regard to jurisdictional claims in published maps and institutional affiliations.

\section{Author details}

'Department of Biochemistry, Biotechnology Research Institute, Kenya Agricultural and Livestock Research Organization, Kikuyu, Kenya. ${ }^{2}$ Department of Biomedical Science and Technology, School of Public Health and Community Development, Maseno University, Private Bag, Maseno, Kenya. ${ }^{3}$ Department of Epidemiology of Microbial Diseases, Yale School of Public Health, New Haven, CT, USA. ${ }^{4}$ Department of Agriculture, School of Agriculture and Food Science, Meru University of Science and Technology, Meru, Kenya. ${ }^{5}$ Centre for Geographic Medicine Research - Coast, Kenya Medical Research Institute, Kilifi, Kenya. ${ }^{6}$ Department of Medical Biochemistry, School of Medicine, Maseno University, Private Bag, Maseno, Kenya.

Received: 5 March 2018 Accepted: 20 June 2018

Published online: 03 July 2018

\section{References}

1. Mattioli RC, Hendrickx G, Wint W, Jannin J, Slingenbergh JFU. Tsetse and trypanosomiasis intervention policies supporting sustainable animal-agricultural development. Food Agric Environ. 2004;2:310-412.

2. Maudlin I, Holmes PH, Miles MA. The trypanosomiasis. Wallingford, UK: CAB International; 2004

3. Chitanga S, Marcotty T, Namangala B, Van den Bossche P, Van Den Abbeele J, Delespaux $V$. High prevalence of drug resistance in animal trypanosomes without a history of drug exposure. PLoS Negl Trop Dis. 2011:5:e1454.

4. Delespaux V, de Koning HP. Drugs and drug resistance in African trypanosomiasis. Drug Resist Update. 2007:10:30-50.

5. Delespaux V, Dinka H, Masumu J, Van den Bossche P, Geerts S. Five-fold increase in Trypanosoma congolense isolates resistant to diminazene aceturate over a seven-year period in eastern Zambia. Drug Resist Updat. 2008;11:205-9.

6. FAO. Drug management and parasite resistance in bovine trypanosomiasis in Africa. In: Geerts S, Holmes PH, editors. Rome: PAAT Technical Scientific Series; 1998.

7. Geerts $\mathrm{S}$, Holmes PH, Eisler MC, Diall O. African bovine trypanosomiasis: the problem of drug resistance. Trends Parasitol. 2001;17:25-8.

8. Holmes P. Tsetse-transmitted trypanosomes - their biology, disease impact and control. J Invertebr Pathol. 2013;112(Suppl.):S11-4.

9. Utz S, Roditi I, Kunz Renggli C, Almeida IC, Acosta-Serrano A, Butikofer P. Trypanosoma congolense procyclins: unmasking cryptic major surface glycoproteins in procyclic forms. Eukaryot Cell. 2006;5:1430-40.

10. Maudlin I, Welburn SC. Maturation of trypanosome infections in tsetse. Exp Parasitol. 1994;79:202-5.

11. Hendry KA, Vickerman K. The requirement for epimastigote attachment during division and metacyclogenesis in Trypanosoma congolense. Parasitol Res. 1988:74:403-8.

12. Peacock L, Cook S, Ferris V, Bailey M, Gibson W. The life cycle of Trypanosoma (Nannomonas) congolense in the tsetse fly. Parasit Vectors. 2012;5:109.

13. Savage AF, Kolev NG, Franklin JB, Vigneron A, Aksoy S, Tschudi C. Transcriptome profiling of Trypanosoma brucei development in the tsetse fly vector Glossina morsitans. PLoS One. 2016;11:e0168877.
14. Koumandou VL, Natesan SK, Sergeenko T, Field MC. The trypanosome transcriptome is remodelled during differentiation but displays limited responsiveness within life stages. BMC Genomics. 2008;9:298.

15. Jensen BC, Sivam D, Kifer CT, Myler PJ, Parsons M. Widespread variation in transcript abundance within and across developmental stages of Trypanosoma brucei. BMC Genomics. 2009;10:482.

16. Queiroz R, Benz C, Fellenberg K, Hoheisel JD, Clayton C. Transcriptome analysis of differentiating trypanosomes reveals the existence of multiple post-transcriptional regulons. BMC Genomics. 2009;10:495.

17. Siegel TN, Hekstra DR, Wang X, Dewell S, Cross GA. Genome-wide analysis of mRNA abundance in two life-cycle stages of Trypanosoma brucei and identification of splicing and polyadenylation sites. Nucleic Acids Res. 2010; 38:4946-57.

18. Veitch NJ, Johnson PC, Trivedi U, Terry S, Wildridge D, MacLeod A. Digital gene expression analysis of two life cycle stages of the human-infective parasite, Trypanosoma brucei gambiense reveals differentially expressed clusters of co-regulated genes. BMC Genomics. 2010;11:124.

19. Urbaniak MD, Guther ML, Ferguson MA. Comparative SILAC proteomic analysis of Trypanosoma brucei bloodstream and procyclic lifecycle stages. PLoS One. 2012;7:e36619.

20. Gunasekera K, Wuthrich D, Braga-Lagache S, Heller M, Ochsenreiter T. Proteome remodelling during development from blood to insect-form Trypanosoma brucei quantified by SILAC and mass spectrometry. BMC Genomics. 2012;13:556

21. Capewell P, Monk S, Ivens A, Macgregor P, Fenn K, Walrad P, et al. Regulation of Trypanosoma brucei total and polysomal mRNA during development within its mammalian host. PLoS One. 2013;8:e67069.

22. Butter F, Bucerius F, Michel M, Cicova Z, Mann M, Janzen CJ. Comparative proteomics of two life cycle stages of stable isotope-labeled Trypanosoma brucei reveals novel components of the parasite's host adaptation machinery. Mol Cell Proteomics. 2013;12:172-9.

23. Greif $G$, Ponce de Leon M, Lamolle G, Rodriguez M, Pineyro D, TavaresMarques $L M$, et al. Transcriptome analysis of the bloodstream stage from the parasite Trypanosoma vivax. BMC Genomics. 2013;14:149.

24. Jackson AP, Goyard S, Xia D, Foth BJ, Sanders M, Wastling JM, et al. Global gene expression profiling through the complete life cycle of Trypanosoma vivax. PLoS Negl Trop Dis. 2015;9:e0003975.

25. Helm JR, Hertz-Fowler C, Aslett M, Berriman M, Sanders M, Quail MA, et al. Analysis of expressed sequence tags from the four main developmental stages of Trypanosoma congolense. Mol Biochem Parasitol. 2009;168:34-42.

26. Eyford BA, Sakurai T, Smith D, Loveless B, Hertz-Fowler C, Donelson JE, et al. Differential protein expression throughout the life cycle of Trypanosoma congolense, a major parasite of cattle in Africa. Mol Biochem Parasitol. 2011; 177:116-25.

27. Tabel $\mathrm{H}$. Activation of the alternative pathway of bovine complement by Trypanosoma congolense. Parasite Immunol. 1982;4:329-35.

28. Otesile EB, Tabel $\mathrm{H}$. Enhanced resistance of highly susceptible Balb/c mice to infection with Trypanosoma congolense after infection and cure. J Parasitol. 1987;73:947-53.

29. Moloo SK. An artificial feeding technique for Glossina. Parasitology. 1971;63: $507-12$

30. Awuoche EO, Weiss BL, Vigneron A, Mireji PO, Aksoy E, Nyambega B, et al. Molecular characterization of tsetse's proboscis and its response to Trypanosoma congolense infection. PLoS Negl Trop Dis. 2017;11:e0006057.

31. Jackson AP, Berry A, Aslett M, Allison HC, Burton P, Vavrova-Anderson J, et al. Antigenic diversity is generated by distinct evolutionary mechanisms in African trypanosome species. Proc Natl Acad Sci USA. 2012;109:3416-21.

32. Aslett M, Aurrecoechea C, Berriman M, Brestelli J, Brunk BP, Carrington M, et al. TriTrypDB: a functional genomic resource for the Trypanosomatidae. Nucleic Acids Res. 2010;38:D457-62.

33. Gibson W. The origins of the trypanosome genome strains Trypanosoma brucei brucei TREU 927, T. b. gambiense DAL 972, T. vivax Y486 and T. congolense IL3000. Parasit Vectors. 2012;5:71.

34. Mortazavi A, Williams BA, McCue $K$, Schaeffer $L$, Wold B. Mapping and quantifying mammalian transcriptomes by RNA-Seq. Nat Meth. 2008:5:621-8.

35. Baggerly KA, Deng L, Morris JS, Aldaz CM. Differential expression in SAGE: accounting for normal between-library variation. Bioinformatics. 2003;19: 1477-83.

36. Robinson MD, McCarthy DJ, Smyth GK. edgeR: a Bioconductor package for differential expression analysis of digital gene expression data. Bioinformatics. 2010;26:139-40. 
37. Gotz S, Garcia-Gomez JM, Terol J, Williams TD, Nagaraj SH, Nueda MJ, et al. High-throughput functional annotation and data mining with the Blast2GO suite. Nucleic Acids Res. 2008:36:3420-35.

38. Conesa A, Gotz S. Blast2GO: a comprehensive suite for functional analysis in plant genomics. Int J Plant Genomics. 2008;2008:619832.

39. Conesa A, Gotz S, Garcia-Gomez JM, Terol J, Talon M, Robles M. Blast2GO: a universal tool for annotation, visualization and analysis in functional genomics research. Bioinformatics. 2005;21:3674-6.

40. Altschul SF, Gish W, Miller W, Myres EW, Lipman DJ. Basic local alignment search tool. J Mol Biol. 1990;215:403-10.

41. Kelly RJ, Vincent DE, Friedberg I. IPRStats: visualization of the functional potential of an InterProScan run. BMC Bioinformatics. 2010;11(Suppl. 1):S13.

42. Jackson AP, Allison HC, Barry JD, Field MC, Hertz-Fowler C, Berriman M. A cell-surface phylome for African trypanosomes. PLoS Negl Trop Dis. 2013;7: e2121.

43. Pierleoni A, Martelli PL, Casadio R. PredGPI: a GPI-anchor predictor. BMC Bioinformatics. 2008;9:392.

44. Poisson G, Chauve C, Chen X, Bergeron A. FragAnchor: a large-scale predictor of glycosylphosphatidylinositol anchors in eukaryote protein sequences by qualitative scoring. Genomics Proteomics Bioinforma. 2007;5: 121-30.

45. Eisenhaber B, Bork P, Eisenhaber F. Prediction of potential GPI-modification sites in proprotein sequences. J Mol Biol. 1999;292:741-58.

46. Krogh A, Larsson B, von Heijne G, Sonnhammer EL. Predicting transmembrane protein topology with a hidden Markov model: application to complete genomes. J Mol Biol. 2001;305:567-80.

47. Petersen TN, Brunak S, Heijne G, Nielsen H. SignalP 4.0: discriminating signal peptides from transmembrane regions. Nat Methods. 2011;8

48. Okuda S, Yamada T, Hamajima M, Itoh M, Katayama T, Bork P, et al. KEGG Atlas mapping for global analysis of metabolic pathways. Nucleic Acids Res. 2008;36:W423-6.

49. Kanehisa M, Goto S, Sato Y, Kawashima M, Furumichi M, Tanabe M. Data, information, knowledge and principle: back to metabolism in KEGG. Nucleic Acids Res. 2014;42:D199-205.

50. Shameer S, Logan-Klumpler FJ, Vinson F, Cottret L, Merlet B, Achcar F, et al. TrypanoCyc: a community-led biochemical pathways database for Trypanosoma brucei. Nucleic Acids Res. 2015;43:D637-44.

51. Pfaffl MW, Tichopad A, Prgomet C, Neuvians TP. Determination of stable housekeeping genes, differentially regulated target genes and sample integrity: BestKeeper - Excel-based tool using pair-wise correlations. Biotechnol Lett. 2004;26:509-15.

52. Savage AF, Cerqueira GC, Regmi S, Wu Y, El Sayed NM, Aksoy S. Transcript expression analysis of putative Trypanosoma brucei GPI-anchored surface proteins during development in the tsetse and mammalian hosts. PLoS Negl Trop Dis. 2012;6:e1708.

53. Schindelin J, Arganda-Carreras I, Frise E, Kaynig V, Longair M, Pietzsch T, et al. Fiji: an open-source platform for biological-image analysis. Nat Methods. 2012;9:676-82.

54. Berger BJ, Dai WW, Wang H, Stark RE, Cerami A. Aromatic amino acid transamination and methionine recycling in trypanosomatids. Proc Natl Acad Sci USA. 1996:93:4126-30

55. Creek DJ, Chokkathukalam A, Jankevics A, Burgess KE, Breitling R, Barrett MP. Stable isotope-assisted metabolomics for network-wide metabolic pathway elucidation. Anal Chem. 2012:84:8442-7.

56. Creek DJ, Mazet M, Achcar F, Anderson J, Kim DH, Kamour R, et al. Probing the metabolic network in bloodstream-form Trypanosoma brucei using untargeted metabolomics with stable isotope labelled glucose. PLoS Pathog. 2015;11:e1004689.

57. Matetovici I, Caljon G, Van Den Abbeele J. Tsetse fly tolerance to T. brucei infection: transcriptome analysis of trypanosome-associated changes in the tsetse fly salivary gland. BMC Genomics. 2016;17:971.

58. Telleria EL, Benoit JB, Zhao X, Savage AF, Regmi S, e Silva TLA, et al. Insights into the trypanosome-host interactions revealed through transcriptomic analysis of parasitized tsetse fly salivary glands. PLoS Negl Trop Dis. 2014;8: e2649.

59. Kolev NG, Ullu E, Tschudi C. The emerging role of RNA-binding proteins in the life cycle of Trypanosoma brucei. Cell Microbiol. 2014;16:482-9.

60. Alves e Silva TL, Savage AF, Aksoy S. Transcript abundance of putative lipid phosphate phosphatases during development of Trypanosoma brucei in the tsetse fly. Am J Trop Med Hyg. 2016;94:890-3.

61. Dean S, Marchetti R, Kirk K, Matthews KR. A surface transporter family conveys the trypanosome differentiation signal. Nature. 2009;459:213-7.
62. Ling AS, Trotter JR, Hendriks EF. A zinc finger protein, TbZC3H20, stabilizes two developmentally regulated mRNAs in trypanosomes. J Biol Chem. 2011;286:20152-62.

63. Kolev NG, Ramey-Butler K, Cross GA, Ullu E, Tschudi C. Developmental progression to infectivity in Trypanosoma brucei triggered by an RNAbinding protein. Science. 2012;338:1352-3.

64. Subota I, Rotureau B, Blisnick T, Ngwabyt S, Durand-Dubief M, Engstler M, et al. ALBA proteins are stage regulated during trypanosome development in the tsetse fly and participate in differentiation. Mol Biol Cell. 2011;22:4205-19.

65. McKerrow JH, Caffrey C, Kelly B, Loke P, Sajid M. Proteases in parasitic diseases. Annu Rev Pathol. 2006;1:497-536.

66. Lalmanach G, Boulange A, Serveau C, Lecaille F, Scharfstein J, Gauthier F, et al. Congopain from Trypanosoma congolense: drug target and vaccine candidate. Biol Chem. 2002;383:739-49.

67. Prain CJ, Ross CA. Trypanosoma congolense: appearance and distribution of variable antigen types during metacyclic differentiation in vitro. Parasitology. 1990;100:107-13.

68. Mony BM, Matthews KR. Assembling the components of the quorum sensing pathway in African trypanosomes. Mol Microbiol. 2015;96:220-32.

69. Makin L, Gluenz E. cAMP signalling in trypanosomatids: role in pathogenesis and as a drug target. Trends Parasitol. 2015;31:373-9.

70. Oberholzer M, Lopez MA, McLelland BT, Hill KL. Social motility in african trypanosomes. PLoS Pathog. 2010;6:e1000739.

71. Oberholzer M, Saada EA, Hill KL. Cyclic AMP regulates social behavior in African trypanosomes. MBio. 2015;6:e01954-14.

72. Saada EA, DeMarco SF, Shimogawa MM, Hill KL. "With a little help from my friends" - social motility in Trypanosoma brucei. PLoS Pathog. 2015;11:e1005272.

73. Salmon D, Vanwalleghem G, Morias Y, Denoeud J, Krumbholz C, Lhomme F, et al. Adenylate cyclases of Trypanosoma brucei inhibit the innate immune response of the host. Science. 2012;337:463-6.

74. Saada EA, Kabututu ZP, Lopez M, Shimogawa MM, Langousis G, Oberholzer $M$, et al. Insect stage-specific receptor adenylate cyclases are localized to distinct subdomains of the Trypanosoma brucei flagellar membrane. Eukaryot Cell. 2014;13:1064-76.

75. Salmon D, Bachmaier S, Krumbholz C, Kador M, Gossmann JA, Uzureau P, et al. Cytokinesis of Trypanosoma brucei bloodstream forms depends on expression of adenylyl cyclases of the ESAG4 or ESAG4-like subfamily. Mo Microbiol. 2012;84:225-42.

76. Lopez MA, Saada EA, Hill KL. Insect stage-specific adenylate cyclases regulate social motility in African trypanosomes. Eukaryot Cell. 2015;14:104-12.

77. Imhof S, Vu XL, Butikofer P, Roditi I. A glycosylation mutant of Trypanosoma brucei links social motility defects in vitro to impaired colonization of tsetse flies in vivo. Eukaryot Cell. 2015;14:588-92.

78. Imhof S, Knusel S, Gunasekera K, Vu XL, Roditi I. Social motility of African trypanosomes is a property of a distinct life-cycle stage that occurs early in tsetse fly transmission. PLoS Pathog. 2014;10:e1004493.

79. Hendriks EF, Robinson DR, Hinkins M, Matthews KR. A novel CCCH protein which modulates differentiation of Trypanosoma brucei to its procyclic form. EMBO J. 2001;20:6700-11.

80. Droll D, Minia I, Fadda A, Singh A, Stewart M, Queiroz R, et al. Post-transcriptional regulation of the trypanosome heat shock response by a zinc finger protein. PLoS Pathog. 2013:9:e1003286.

81. Brindley DN, Pilquil C, Sariahmetoglu M, Reue K. Phosphatidate degradation: phosphatidate phosphatases (lipins) and lipid phosphate phosphatases. Biochim Biophys Acta. 2009;1791:956-61.

82. Pascual F, Carman GM. Phosphatidate phosphatase, a key regulator of lipid homeostasis. Biochim Biophys Acta. 2013;1831:514-22.

83. Butikofer $P$, Vassella $E$, Boschung M, Renggli CK, Brun R, Pearson TW, et al. Glycosylphosphatidylinositol-anchored surface molecules of Trypanosoma congolense insect forms are developmentally regulated in the tsetse fly. Mol Biochem Parasitol. 2002;119:7-16.

84. Sakurai T, Sugimoto C, Inoue N. Identification and molecular characterization of a novel stage-specific surface protein of Trypanosoma congolense epimastigotes. Mol Biochem Parasitol. 2008;161:1-11.

85. Donelson JE, Hill KL, El-Sayed NM. Multiple mechanisms of immune evasion by African trypanosomes. Mol Biochem Parasitol. 1998;91:51-66.

86. Alsford S, Eckert S, Baker N, Glover L, Sanchez-Flores A, Leung KF, et al. High-throughput decoding of antitrypanosomal drug efficacy and resistance. Nature. 2012;482:232-6.

87. Lane-Serff H, MacGregor P, Peacock L, Macleod OJ, Kay C, Gibson W, et al. Evolutionary diversification of the trypanosome haptoglobin-haemoglobin receptor from an ancestral haemoglobin receptor. Elife. 2016;5 
88. Higgins MK, Lane-Serff H, MacGregor P, Carrington M. A receptor's tale: an eon in the life of a trypanosome receptor. PLoS Pathog. 2017;13

89. Vanhollebeke B, De Muylder G, Nielsen MJ, Pays A, Tebabi P, Dieu M, et al. A haptoglobin-hemoglobin receptor conveys innate immunity to Trypanosoma brucei in humans. Science. 2008;320:677-81.

90. Thevenaz P, Hecker H. Distribution and attachment of Typanosoma (Nannomonas) congolense in the proximal part of the proboscis of Glossina morsitans morsitans. Acta Trop. 1980;37:163-75.

91. Nantulya VM, Doyle JJ, Jenni L. Studies on Trypanosoma (Nannomonas) congolense. I. On the morphological appearance of the parasite in the mouse. Acta Trop. 1978;35:329-37.

92. Nantulya VM, Doyle JJ, Jenni L. Studies on Trypanosoma (Nannomonas) congolense. IV. Experimental immunization of mice against tsetse fly challenge. Parasitology. 1980;80:133-7.

93. Nantulya VM. Immunological approaches to the control of animal trypanosomiasis. Parasitol Today. 1986;2:168-73.

94. Schwenk R, Lumsden JM, Rein LE, Juompan L, Kester KE, Heppner DG, et al. Immunization with the RTS,S/AS malaria vaccine induces IFN-gamma(+)CD4 $T$ cells that recognize only discrete regions of the circumsporozoite protein and these specificities are maintained following booster immunizations and challenge. Vaccine. 2011;29:8847-54.

95. Zavala F, Tam JP, Hollingdale MR, Cochrane AH, Quakyi I, Nussenzweig RS, et al. Rationale for development of a synthetic vaccine against Plasmodium falciparum malaria. Science. 1985;228:1436-40.

96. Chaturvedi N, Bharti PK, Tiwari A, Singh N. Strategies \& recent development of transmission-blocking vaccines against Plasmodium falciparum. Indian J Med Res. 2016;143:696-711.

97. Aksoy S, Maudlin I, Dale C, Robinson AS, O'Neill SL. Prospects for control of African trypanosomiasis by tsetse vector manipulation. Trends Parasitol. 2001;17:29-35.

98. Logan-Klumpler FJ, De Silva N, Boehme U, Rogers MB, Velarde G, McQuillan JA, et al. GeneDB - an annotation database for pathogens. Nucleic Acids Res. 2012:40:D98-108.

99. McCarthy DJ, Chen Y, Smyth GK. Differential expression analysis of multifactor RNA-Seq experiments with respect to biological variation. Nucleic Acids Res. 2012;40:4288-97.

\section{Ready to submit your research? Choose BMC and benefit from:}

- fast, convenient online submission

- thorough peer review by experienced researchers in your field

- rapid publication on acceptance

- support for research data, including large and complex data types

- gold Open Access which fosters wider collaboration and increased citations

- maximum visibility for your research: over $100 \mathrm{M}$ website views per year

At BMC, research is always in progress.

Learn more biomedcentral.com/submissions 